\title{
A linear elastic - brittle interface model: Application for the onset and propagation of a fibre-matrix interface crack under biaxial transverse loads
}

\author{
Vladislav Mantič • Luis Távara • Antonio \\ Blázquez • Enrique Graciani · Federico \\ París.
}

Received: date / Accepted: date

\begin{abstract}
A new linear elastic and perfectly brittle interface model for mixed mode is presented and analysed. In this model, the interface is represented by a continuous distribution of springs which simulates the presence of a thin elastic layer. The constitutive law for the continuous distribution of normal and tangential initially-linear-elastic springs takes into account possible frictionless elastic contact between adherents once a portion of the interface is broken. A perfectly brittle failure criterion is employed for the springs, which enables the study of crack onset and propagation. This interface failure criterion takes into account the variation of the interface fracture toughness with the fracture mode mixity. A unified way to represent several phenomenological both energy and stress based failure criteria is introduced. A proof relating the energy release rate and tractions at an interface point (not necessarily a crack tip point) is introduced for this interface model by adapting Irwin's crack closure technique for the first time. The main advantages of the present interface model are its simplicity, robustness and computational efficiency, even in the presence of snap-back and snap-through instabilities, when the so-called sequentially linear (elastic) analysis is applied. This model is applied here in order to study crack onset and propagation at the fibre-matrix interface in a composite under tensile/compressive remote biaxial transverse loads. Firstly, this model is used to obtain analytical predictions about interface crack onset, while investigating a single fibre embedded in a matrix which is subjected to uniform remote transverse loads. Then, numerical results provided by a $2 \mathrm{D}$ boundary element analysis show that a fibre-matrix interface failure is initiated by the onset of a finite debond in the neighbourhood of the interface point where the failure criterion is first reached (under increasing proportional load); this debond further propagates along the interface in mixed mode or even, in some configurations, with the crack tip under compression. The analytical predictions of the debond onset
\end{abstract}

V. Mantič · L.Távara $\bowtie$ A. Blázquez · E. Graciani · F.París Grupo de Elasticidad y Resistencia de Materiales,

Escuela Técnica Superior de Ingeniería, Universidad de Sevilla,

Camino de los Descubrimientos s/n, ES-41092 Sevilla, Spain

Tel.: +34954487300

Fax: +34954461637

E-mail: ltavara@etsi.us.es 
position and associated critical load are used for several parametric studies of the influence of load biaxiality, fracture-mode sensitivity and brittleness number, and for checking the computational procedure implemented.

Keywords Debonding · Fracture toughness · Interfacial strength · Failure criteria $\cdot$ Transverse cracking $\cdot$ Adhesive contact

\section{Introduction}

Matrix (or interfibre) failure in composite unidirectional laminates subjected to loads transverse to the fibres is often initiated by the debonding of some fibres (Hull and Clyne 1996; Zhang et al 1997; Varna et al 1997; París et al 2007; Correa et al 2008b,a). The problem of an elastic circular cylindrical inclusion (fibre) embedded in an elastic matrix, with or without a partial debond at its interface, subjected to uniaxial tensile/compressive loads, has been studied a great deal in the past. An extensive review of these studies can be found in (París et al 2007; Mantič 2009; Távara et al 2011). In the current investigation, debond onset and propagation along the interface of an isolated fibre embedded in an elastic matrix subjected to remote biaxial transverse loads is studied, cf. (París et al 2003; Mantič and García 2012; Correa et al 2013). The aim of the study is to obtain, among other results, failure curves predicting the critical loads which cause the fibre-matrix interface failure. The results presented may contribute to understanding the mechanisms of damage initiation in unidirectional composite laminas under transverse loads.

In many practical situations, the behavior of (adhesively) bonded solids, referred to as adherents, has been described by modelling a thin (adhesive) elastic layer, also called interphase, by an interface between adherents with certain characteristics. As was demonstrated by Benveniste and Miloh (2001) using an asymptotic approach, there are seven types of these interface conditions corresponding to either soft or stiff thin layers in relation to an average stiffness of adherents. The most commonly used type of interface, called spring type interface in their classification, corresponds to a relatively soft layer with constant stresses across its thickness. This classical model, usually referred to as linear-elastic interface, weak interface or imperfect interface, can be considered as a continuous distribution of linear-elastic springs with appropriate stiffness parameters (Goland and Reissner 1944; Erdogan 1997; Geymonat et al 1999; Benveniste and Miloh 2001; Lenci 2001; Hashin 2002). Note that imperfect interface sometimes refers to a more general relationship between displacement and tractions of adherents along the interface, cf. (Benveniste and Miloh 2001; Hashin 2002).

As proposed recently by several authors (Caporale et al 2006; Bennati et al 2009; Carpinteri et al 2009; Távara et al 2010, 2011; Cornetti et al 2012; Weißgraeber and Becker 2013), a practical way to describe debonding or delamination processes is to enrich this classical model by including strength and/or fracture parameters and an associated failure criteria. Such a model can also be considered as a limit "non-smooth case" for some (nonlinear) cohesive zone models (CZMs) (Bialas and Mróz 2005b; Valoroso and Champaney 2006; Cornetti et al 2015). In fact, this kind of interface model with a linear traction-separation relationship up to a maximum value of separation was originally suggested by Prandtl (1933) and Mott (1948); for further historical references to this model see a discussion in 
Entov and Salganik (1968). A new energetic formulation for this model (with an elasto-plastic hardening interface) based on the minimization of the total potential energy plus dissipated energy for each load increment has been recently developed in Panagiotopoulos et al (2013); Roubíček et al (2013); Vodička et al (2014).

With reference to the specific problem of fibres embedded in a matrix, many authors consider that an appropriate way to describe the physical nature and mechanical behavior of the fibre-matrix interface is through the application of this elastic interface model, see Távara et al (2011); Hashin (2002) and references therein. An analytical closed-form solution of a single circular inclusion problem, assuming an undamaged linear-elastic interface under remote tension, was deduced by Gao (1995). A generalization of this solution was later presented by Bigoni et al (1998). Mogilevskaya and Crouch (2002) numerically solved the problem of an infinite, isotropic, elastic plane containing a large number of randomly distributed circular elastic inclusions with spring-like interface conditions. Later, Caporale et al (2006) applied a linear elastic - (perfectly) brittle law, using normal and shear interface-strength criteria and the 3D finite element method (FEM) to determine curves of macro-strains corresponding to the initiation of the interfacial debonding.

Following similar ideas, other authors have applied different CZMs to model the fibre-matrix debond, a few of them being mentioned below. Levy and co-workers in a series of studies, see Xie and Levy (2007) and references therein, carried out parametric studies on the stability of the phenomenon of circular-inclusion decohesion under biaxial loading applying a CZM. Carpinteri et al (2005) used a CZM to study the instability phenomena in fibrous metal matrix composites through FEM. Han et al (2006) used a softening decohesion model to study the initiation and propagation of debonds in several single and two fibre configurations using the boundary element method (BEM). Recently, Ngo et al (2010) used a new potential-based CZM to study the inclusion-matrix debonding in an integrated approach involving micromechanics, and Kushch et al (2011) used a bi-linear CZM to simulate progressive debonding in multi-fibre models for a composite showing the formation of debond clusters.

An alternative analytical approach based on a coupled stress and energy criterion (Mantič 2009; Leguillon 2002) and the classical open model of interface cracks (Mantič et al 2006) has recently been applied by Mantič and García (2012) to describe the initiation and propagation of a fibre-matrix interface crack under biaxial loads.

In the present investigation, the linear elastic - (perfectly) brittle interface model (LEBIM) originally proposed by Prandtl (1933) and Mott (1948) and further developed by Távara et al $(2010,2011)$ is employed because of its simplicity, robustness and computational efficiency. This model is enhanced here in order to cover also interface fracture due to shear under compression, by extending the range of variation of the interface fracture toughness with the fracture mode mixity, and by considering the possibility of frictionless elastic contact at broken portions of the interface.

The scope of application of the present LEBIM has two obvious limitations related to the possible presence of a softening zone ahead of the crack tip and a friction contact zone between crack faces. The LEBIM is applicable if the energies dissipated in the softening and/or contact zones are sufficiently small in comparison with the energy required to interface fracture. This essentially means that the softening and contact zone are sufficiently small in comparison with the small- 
est characteristic length of the specimen (e.g. crack length or an adjacent layer thickness), such situations being usually referred to as the small-scale cohesive zone length (Wang 2013) or small-scale contact zone length. Moreover in the case of large contact zones between crack faces this could mean that either the friction coefficient or contact compressions are sufficiently low. It appears that these conditions are verified, e.g., in the interlaminar fracture toughness test of composite laminates where an excellent agreement between the experimental results and numerical predictions by the LEBIM is achieved (Távara et al 2010).

This new LEBIM is used together with Gao's analytical solution (Gao 1995; Távara et al 2011) for evaluating the failure curve of a single fibre under biaxial loads, which may provide an approximation of the corresponding failure curve for diluted fibre packing (low fibre volume fraction). The LEBIM is also implemented in a $2 \mathrm{D}$ collocational BEM code, used to study the debond initiation and propagation in the present investigation; this will also allow the problem of debond initiation and propagation to be solved accurately and efficiently for dense fibre packing (high fibre volume fraction) including many fibres in forthcoming studies, see Távara (2010); Távara et al (2013) for some preliminary results.

The LEBIM with the extended interface failure criterion is presented in Section 2. In Section 3, the problem of a circular inclusion under a remote biaxial transverse loading is defined and Gao's analytical solution is reviewed. Both the analytical and numerical BEM procedures for fibre-matrix debond modelling are described in Section 4. Finally, the influence of the load biaxiality parameter $\chi$ and three dimensionless governing parameters (the ratio of the interface shear and normal stiffnesses $\xi$, the fracture mode-sensitivity parameter $\lambda$ and the brittleness number $\gamma$ ) on the position of debond initiation, on the value of critical biaxial transverse load and on further debond propagation is studied and discussed in Section 5. For the sake of simplicity, once the variable dependencies are defined, the functions are usually represented as functions of only a few of the most relevant arguments or (non-arguments).

\section{Linear elastic - (perfectly) brittle interface model (LEBIM)}

Newly enhanced constitutive law and failure criterion for the LEBIM, cf. (Távara et al 2010, 2011; Távara 2010), are introduced in Section 2.1. Although this interface model was originally conceived in order to represent a thin adhesive layer $h>0$ (see Section 2.3), it can be defined and applied to simulate debonding mechanisms of bimaterial systems where, strictly speaking, there is no additional third material (usually referred to as interphase) between the bonded materials, as may occur in the present case of fibre-matrix interface in a real composite. Therefore, the continuous distribution of springs in the LEBIM has zero thickness.

Stress and energy based failure criteria have coexisted in Solid Mechanics for a long time both have been used in different problems and for different purposes (see Section 2.4). Typically, the former are applied at concentrations of stresses in order to predict failure initiation, whereas the latter are used to predict the propagation of existing cracks. Nevertheless, in the current (may be the most straightforward) formulation of constitutive law and failure criterion for LEBIM, with no traction singularity at a crack tip, these two criteria somewhat surprisingly become equivalent. It appears that this quite surprising observation has been repeatedly 
rediscovered by many researchers during the last 50 years showing (apparently independently), in different ways that the ERR at a crack tip (actually the endpoint of an undamaged interface zone) is determined by the interface tractions at this very crack tip point (Entov and Salganik 1968; Fernlund and Spelt 1991; Krenk 1992; Bank-Sills and Salganik 1994; Erdogan 1997; Lenci 2001; Bruno and Greco 2001; Shahin and Taheri 2008; Carpinteri et al 2009). Actually, as will be shown in Section 2.2, ERR can be defined for breaking of any small undamaged portion of the linear elastic-brittle interface (not necessarily the crack tip point) and is determined by tractions at the point where the breakage initiates and just at the moment before the breakage occurs. In this sense, although the LEBIM will be introduced below, using an energy failure criterion, it could also be defined in terms of tractions or even relative displacements at the interface point under scrutiny.

\subsection{Constitutive law of the spring distribution}

The continuous distribution of springs is governed by a constitutive law which prescribes the relation between tractions and relative displacements at the interface, as illustrated in Fig. 1, cf. Bruno et al (2003).

\section{Linear elastic - (perfectly) brittle interface}

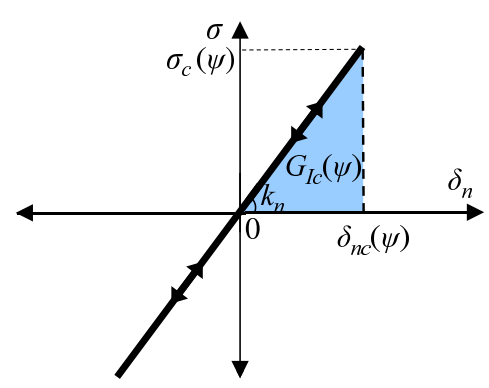

(a)

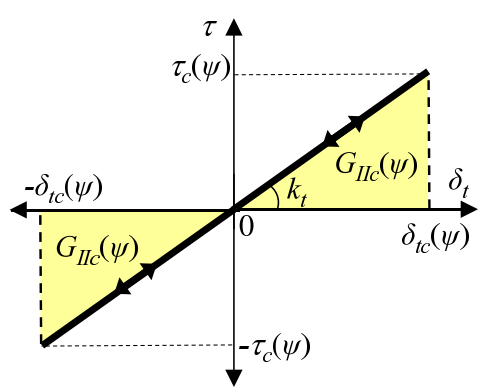

(b)

Broken interface

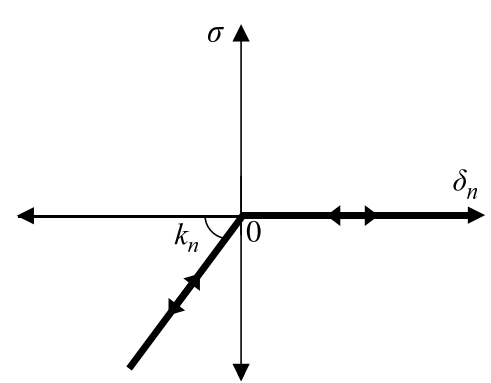

(c)

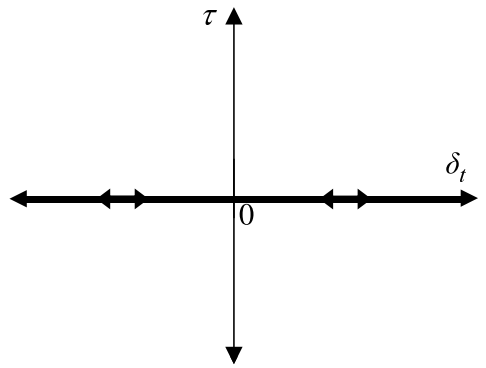

(d)

Fig. 1 Linear elastic - (perfectly) brittle law in the undamaged interface in (a) the normal direction and (b) tangential direction, and in the broken interface in (c) the normal direction and (d) tangential direction. 
The following simple linear-elastic law relates tractions and relative displacements at an interface point $x$ located along an undamaged part of the interface, Fig. 1(a) and (b):

$$
\begin{aligned}
& \text { Linear Elastic } \\
& \text { Interface }
\end{aligned}\left\{\begin{array}{l}
\sigma(x)=k_{n} \delta_{n}(x), \\
\tau(x)=k_{t} \delta_{t}(x),
\end{array} \text { for } \quad G(x)<G_{c}(\psi(x))\right.
$$

where $\sigma(x)$ and $\tau(x)$ are the normal and shear tractions at $x, \delta_{n}(x)$ and $\delta_{t}(x)$ are the normal (opening) and tangential (sliding) relative displacements between opposite interface points, and $k_{n}$ and $k_{t}$ denote the normal and tangential stiffness of the spring distribution, respectively.

The interface failure criterion adopted here is defined in terms of Energy Release Rate (ERR) $G$ and interface fracture energy (also known as fracture toughness) $G_{c}$. As discussed above, the ERR in the LEBIM is given by the stored elastic strain energy (per unit area) in an unbroken "interface spring" (infinitesimal interface segment) at a point $x$, see Section 2.2 for proof. Thus, the ERR of a mixed mode crack in a linear elastic interface can be computed as, cf. (Távara et al 2011, 2010):

$$
G(x)=G_{I}(x)+G_{I I}(x)
$$

with

$$
\begin{aligned}
G_{I}(x) & =\frac{\langle\sigma(x)\rangle_{+}\left\langle\delta_{n}(x)\right\rangle_{+}}{2}=\frac{\langle\sigma(x)\rangle_{+}^{2}}{2 k_{n}}=\frac{k_{n}\left\langle\delta_{n}(x)\right\rangle_{+}^{2}}{2} \\
G_{I I}(x) & =\frac{\tau(x) \delta_{t}(x)}{2}=\frac{\tau^{2}(x)}{2 k_{t}}=\frac{k_{t} \delta_{t}^{2}(x)}{2}
\end{aligned}
$$

verifying that $G_{I}(x)=0$ for $\sigma(x) \leq 0$. The positive and negative parts of a real number $\delta$, used in the present study, are defined as $\langle\delta\rangle_{ \pm}=\frac{1}{2}(\delta \pm|\delta|) \cdot\langle\cdot\rangle_{+}$and which is also referred to as Macaulay brackets or ramp function.

An extension of the energetic fracture-mode-mixity-angle $\psi_{G}$, introduced in (Távara et al 2011,2010) by the relation $\tan ^{2} \psi_{G}=G_{I I} / G_{I}$ for $G_{I}>0$ and $0 \leq \psi_{G} \leq \frac{\pi}{2}$, which will cover also an interface under compression with $\sigma<0$, can be defined by

$$
\tan \psi=\sqrt{\xi^{-1}} \tan \psi_{\sigma}=\sqrt{\xi} \tan \psi_{u}, \quad-\pi \leq \psi, \psi_{\sigma}, \psi_{u} \leq \pi,
$$

where

$$
\xi=\frac{k_{t}}{k_{n}},
$$

and $\tan \psi_{\sigma}=\tau / \sigma$ and $\tan \psi_{u}=\delta_{t} / \delta_{n} . \psi_{\sigma}$ and $\psi_{u}$ being the stress and relative displacement based fracture-mode-mixity angles, respectively. Notice that $\psi=\psi_{G}$ for $\sigma>0$, and that the absolute value of the tangent of $\psi$ is given by the geometric mean of tangents of $\psi_{\sigma}$ and $\psi_{u}$, i.e. $|\tan \psi|=\sqrt{\tan \psi_{\sigma} \tan \psi_{u}}$. It is easy to check that although these angles $\left(\psi, \psi_{\sigma}\right.$ and $\left.\psi_{u}\right)$ are in general different, they coincide for particular values of $0, \pm \frac{\pi}{2}$ and $\pm \pi$.

According to the interface failure criterion in (1), an interface point, not necessarily a crack tip point, breaks when the ERR, $G$, in (2) reaches the fracture energy, $G_{c}$, which depends on the fracture mode mixity, i.e. $G=G_{c}(\psi)$, where

$$
G_{c}(\psi)=G_{I c}(\psi)+G_{I I c}(\psi),
$$


which in view of (2) can be expressed as, cf. Fig. 1(a) and (b),

$$
\begin{aligned}
G_{I c}(\psi) & =\frac{\left\langle\sigma_{c}(\psi)\right\rangle_{+}\left\langle\delta_{n c}(\psi)\right\rangle_{+}}{2}=\frac{\left\langle\sigma_{c}(\psi)\right\rangle_{+}^{2}}{2 k_{n}}=\frac{k_{n}\left\langle\delta_{n c}(\psi)\right\rangle_{+}^{2}}{2}, \\
G_{I I c}(\psi) & =\frac{\tau_{c}(\psi) \delta_{t c}(\psi)}{2}=\frac{\tau_{c}^{2}(\psi)}{2 k_{t}}=\frac{k_{t} \delta_{t c}^{2}(\psi)}{2}
\end{aligned}
$$

The above formulas define the critical normal and shear tractions $\sigma_{c}(\psi)$ and $\tau_{c}(\psi)$ and also the corresponding critical relative displacements $\delta_{n c}(\psi)$ and $\delta_{t c}(\psi)$ as functions of the fracture-mode-mixity angle $\psi$, veryfing $\sigma_{c}(\psi)=k_{n} \delta_{n c}(\psi)$ and $\tau_{c}(\psi)=k_{t} \delta_{t c}(\psi)$. Different critical values of these variables may be obtained at different interface points, due to the fact that $\psi$ can vary along the interface.

Let $\bar{G}_{I c}=G_{c}(0)>0$ denote the interface fracture toughness in pure mode I $(\psi=0)$ and $\bar{\sigma}_{c}=\sigma_{c}(0)>0$ the critical interface normal stress in pure mode I (interfacial tensile strength). Similarly, $\bar{G}_{I I c}=G_{c}\left(\frac{\pi}{2}\right)>0$ and $\bar{\tau}_{c}=\tau_{c}\left(\frac{\pi}{2}\right)>0$. Then, from (5b) and (5c), cf. Távara et al (2011, 2010),

$$
\bar{G}_{I c}=\frac{\bar{\sigma}_{c}^{2}}{2 k_{n}} \quad \text { and } \quad \bar{G}_{I I c}=\frac{\bar{\tau}_{c}^{2}}{2 k_{t}} \text {. }
$$

Using these definitions we can express the fracture energies in terms of $\bar{G}_{I c}$ and dimensionless functions, e.g., $\hat{G}_{c}(\psi)\left(\hat{G}_{c}(0)=1\right)$, characterizing their variations with fracture mode mixity,

$$
G_{c}(\psi)=\bar{G}_{I c} \hat{G}_{c}(\psi), \quad G_{I c}(\psi)=\bar{G}_{I c} \hat{G}_{I c}(\psi) \quad \text { and } \quad G_{I I c}(\psi)=\bar{G}_{I c} \hat{G}_{I I c}(\psi) .
$$

Similarly we can express the critical normal and shear tractions in terms of $\bar{\sigma}_{c}$ and dimensionless functions $\hat{\sigma}_{c}(\psi)\left(\hat{\sigma}_{c}(0)=1\right)$ and $\hat{\tau}_{c}(\psi)$ as

$$
\sigma_{c}(\psi)=\bar{\sigma}_{c} \hat{\sigma}_{c}(\psi) \quad \text { and } \quad \tau_{c}(\psi)=\bar{\sigma}_{c} \hat{\tau}_{c}(\psi)
$$

After some algebraic manipulations, we can obtain from (3-8) the following general relations between dimensionless fracture energy and critical tractions:

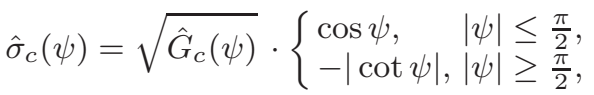

$$
\begin{aligned}
& \hat{\tau}_{c}(\psi)=\sqrt{\xi} \sqrt{\hat{G}_{c}(\psi)} \cdot\left\{\begin{array}{l}
\sin \psi,|\psi| \leq \frac{\pi}{2}, \\
\operatorname{sign} \psi,|\psi| \geq \frac{\pi}{2} .
\end{array}\right.
\end{aligned}
$$

The switch in these expressions for $|\psi| \lessgtr \frac{\pi}{2}$, and equivalently for $\sigma \gtrless 0$, is due to the fact that $G_{I}=0$ in (2) and also $G_{I c}=0$ in (5) for $|\psi| \geq \frac{\pi}{2}$.

The following implicit equation for the failure curve in the plane of dimensionless tractions (8) is deduced from (9):

$$
\hat{\sigma}_{c}^{2}+\xi^{-1} \hat{\tau}_{c}^{2}=\hat{G}_{c}(\psi) \cdot\left\{\begin{array}{ll}
1, & \hat{\sigma}_{c} \geq 0, \\
\csc ^{2} \psi, & \hat{\sigma}_{c} \leq 0,
\end{array} \quad \text { with } \quad \psi=\arctan \left(\sqrt{\xi} \hat{\sigma}_{c}, \hat{\tau}_{c}\right) .\right.
$$

The (non-trivial!) equivalency of the ERR and the tractions on one hand, and the fracture energy and critical tractions on the other hand, at a particular unbroken interface-point, is represented by the relations in (2) and (5), respectively. This equivalence is also evidenced by (9). In view of the relationship (6), this equivalence implies that the failure criterion in the LEBIM (and actually, as will 
be seen, the whole LEBIM) is defined by four independent parameters: two physical quantities with dimension, one dimensionless quantity and one dimensionless function of a mode mixity angle. For example, a typical energy based set of governing parameters could be $\bar{G}_{I c}, \bar{\sigma}_{c}, \xi$ and $\hat{G}_{c}(\psi)$, whereas a stress based set could include $\bar{\sigma}_{c}, k_{n}, \xi$ and $\hat{\sigma}_{c}(\psi)$.

In view of the above, the energy-based failure-criterion in (1) can be written in terms of either the traction modulus or relative displacement modulus at $x$, $t(x)=\sqrt{\sigma^{2}(x)+\tau^{2}(x)}$ or $\delta(x)=\sqrt{\delta_{n}^{2}(x)+\delta_{t}^{2}(x)}$, respectively. Then, the interface breaks at $x$ when these moduli reach their critical values:

$$
t_{c}(\psi)=\sqrt{\sigma_{c}^{2}(\psi)+\tau_{c}^{2}(\psi)} \text { or } \delta_{c}(\psi)=\sqrt{\delta_{n c}^{2}(\psi)+\delta_{t c}^{2}(\psi)},
$$

i.e. $t(x)=t_{c}(\psi(x))$ and $\delta(x)=\delta_{c}(\psi(x))$. Obviously, different critical values for these moduli may be obtained at different interface points, due to the fact that $\psi$ can vary along the interface.

After reaching this failure criterion, the damaged interface is considered free of stresses, unless contact appears between both sides of the damaged interface. In this case, it is assumed here, for the sake of simplicity, that the interface retains its original normal stiffness (see Section 2.3), although other values for this stiffness could be chosen if suitable. Even infinite stiffness could be considered as in Caporale et al (2006); Panagiotopoulos et al (2013); Roubíček et al (2013); Vodička et al (2014). Therefore, once the interface is broken, the following non-linear constitutive law is considered at an interface point $x$, Fig. 1(c) and (d):

$$
\begin{aligned}
& \text { Broken } \\
& \text { Interface }
\end{aligned}\left\{\begin{array}{l}
\sigma(x)=k_{n}\left\langle\delta_{n}(x)\right\rangle_{-}, \\
\tau(x)=0 .
\end{array}\right.
$$

In order to make the Model more simple, only frictionless contact is considered here, although for situations with relatively large contact zones and high friction coefficient under relevant compressions, friction could be included, e.g., following either Bialas and Mróz (2005a,b), Graciani et al (2005), or Raous (2011) and Kšiñan et al (2014).

\subsection{ERR in LEBIM evaluated by the Crack Closure Technique}

Although several proofs for the formulae (2b) and (2c) for ERR of a crack at a linear elastic interface have (apparently independently) been published in the past in Entov and Salganik (1968); Fernlund and Spelt (1991); Krenk (1992); Erdogan (1997); Lenci (2001); Bruno and Greco (2001); Shahin and Taheri (2008); Carpinteri et al (2009), see also Bank-Sills and Salganik (1994), the present authors believe that the mechanical interpretation, (given below), of Irwin's Crack Closure Technique (Irwin 1957) applied to any unbroken point of this interface, given below, still deserves to be presented.

Let us consider two linear, elastic solids (adherents) $A$ and $B$ bonded along a straight interface, located at the $x$-axis. For the sake of simplicity, we will consider the mode I situation assuming that only normal stresses can be transferred across the interface. Fig. 2 illustrates three states for these adherents and the linear elastic interface: the stress-free state 0 before any load is applied, the state 1 with a load applied and with no spring broken and, finally, the state 2 with a portion of the 
interface of a small length $\Delta a>0$ broken due to a sufficiently highly applied load. Here, for the sake of clarity, a certain initial and constant length $h>0$ of stress-free springs is indicated, although theoretically $h$ can vanish $\left(h \rightarrow 0_{+}\right)$. In state 2 , the stress free parts of the broken springs of lengths $h^{\prime}>0$ and $h^{\prime \prime}>0$, with $h^{\prime}+h^{\prime \prime}=h$, are indicated along the crack length $\Delta a$ on both crack sides, as well. The distance between adherents at a point $x$ in a state $s=0,1$ and 2 is denoted by $\delta_{n}^{(s)}(x)+h$, with $\delta_{n}^{(0)}(x)=0 . \delta_{n}^{(s)}(x)(s=1,2)$ along the bonded parts and represents the elongation/shrinkage of the stretched or compressed springs, whereas $\delta_{n}^{(2)}(x)$ along the broken part of the interface $\Delta a>0$, represents the distance between the two free extremes of the broken springs .

Contrary to classical Fracture Mechanics, which corresponds to perfect (infinitely stiff) interfaces, in a linear elastic interface the breakage of a previously undamaged zone is possible, e.g., at a stress concentration, because the associated ERR is positive, $G_{I}>0$, and can be calculated as shown below by adapting Irwin's Crack Closure Technique to the present case.

Let $U^{(s)}$, for a state $s$, denote the total strain energy in the system under consideration (adherents and linear elastic interface $\equiv$ spring distribution) which is subjected to a load under the so-called displacement control, which means that during a breakage of the interface (from the state 1 to 2 ) no work is done by the external load. Then, the ERR is evaluated as follows, employing the linear elastic behaviour of adherents and interface,

$$
\begin{aligned}
G_{I}\left(x_{0}\right) & =\lim _{\Delta a \rightarrow 0}-\frac{U^{(2)}-U^{(1)}}{\Delta a}=\lim _{\Delta a \rightarrow 0} \frac{1}{\Delta a} \int_{\Delta a} \frac{1}{2} \sigma^{(1)}(x) \delta_{n}^{(2)}(x) d x \text { mean } \underline{=}=\begin{array}{c}
\text { theorem } \\
\text { continuity of displacement } \\
\text { variations from state 2 to 1 }
\end{array}=\frac{1}{2} \sigma^{(1)}\left(x_{0}\right) \delta_{n}^{(1)}\left(x_{0}\right),
\end{aligned}
$$

where the first mean value theorem for integrals has been applied, with $x^{\prime}$ being a point in the broken zone of length $\Delta a$ (cf. Carpinteri et al (2009)), and the continuity of displacement variations between states 2 and 1 for $\Delta a \rightarrow 0$ has been assumed, i.e. $\lim _{\Delta a \rightarrow 0} \delta_{n}^{(2)}(x)=\delta_{n}^{(1)}(x)$ at any point $x$ on the interface. The last term $\frac{1}{2} \sigma^{(1)}\left(x_{0}\right) \delta_{n}^{(1)}\left(x_{0}\right)$ represents the energy per unit area stored in the first spring where breakage begins at a position $x_{0}$.

Actually, the proof can be followed considering $h=0$, but here we have preferred to assume $h>0$, for the sake of illustration.

Adapting this procedure to a crack of initial length $a>0$ advancing by $\Delta a \rightarrow 0$ is evident, we just replace $\delta_{n}^{(2)}(x)$ in (13) by $\delta_{n}^{(1)}(x-\Delta a)$, assuming the original crack is advancing at its right tip. But as we have shown, ERR can be defined for any point on a linear elastic interface, not necessarily at the crack tip.

Notice that Irwins classical Crack Closure Technique corresponds to the limit case of an infinitely stiff layer in the normal direction. The key difference between both procedures is that, although the distance between adherents $A$ and $B$ at a point $x$ in state 2 is $\delta_{n}^{(2)}(x)+h(h \geq 0)$ which ends up with value $\delta_{n}^{(1)}(x)+h$ in state 1 , the relative displacement suffered by the (free) extremes of broken springs at $x$ from state 2 to state 1 is $\delta_{n}^{(2)}(x)$, and not $\delta_{n}^{(2)}(x)-\delta_{n}^{(1)}(x)$, which one 

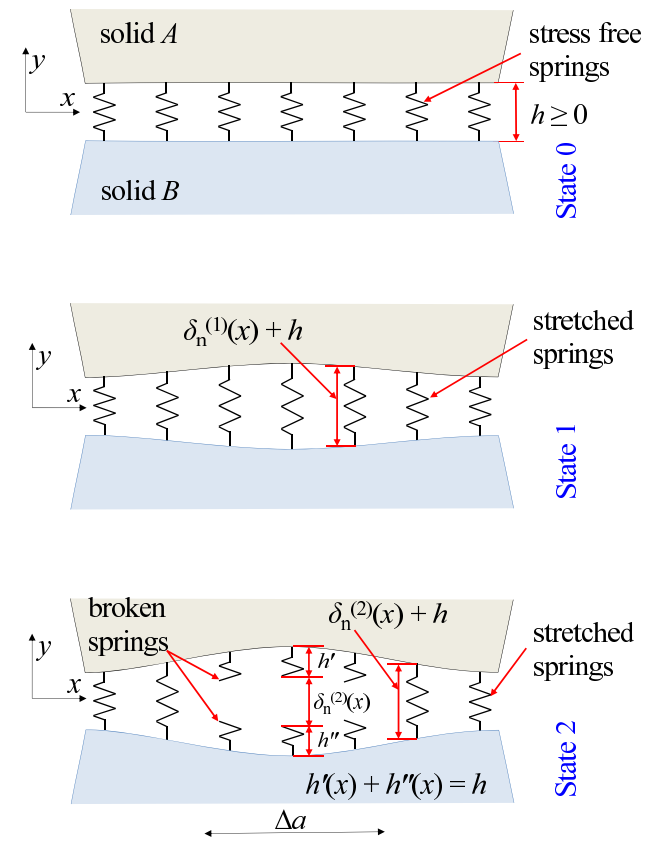

Fig. 2 VCCT for LEBIM (Mode I) applied at a maximum-tension point.

could erroneously think. Actually, $\delta_{n}^{(2)}(x)-\delta_{n}^{(1)}(x)$ represents the variation of the distance between adherents.

The expression of ERR in mode II, $G_{I I}$, can be similarly obtained replacing $\sigma^{(1)}(x) \delta_{n}^{(2)}(x)$ in (13) by $\tau^{(1)}(x) \delta_{t}^{(2)}(x)$. Obviously in the stress free state 0 the separation of opposite interface points in the tangential direction is zero.

Although tractions along a linear elastic interface are bounded, their derivatives are unbounded, having logarithmic singularities at crack tips (Erdogan 1997; Antipov et al 2001; Lenci 2001; Mishuris and Kuhn 2001), cf. Távara et al (2010). Thus, the (local) maximum values of tractions at crack tips are generally highly ill-defined, and consequently to extract accurately these maximum values, which determine ERRs at these crack tips in view of (13), from numerical methods such as FEM or BEM, strongly refined meshes may be required.

It is noteworthy that in the case of a linear elastic interface an elastic stress field in the neighbourhood of an interface crack tip has a logarithmic singularity with unbounded stresses inside the domain close to the crack tip. Thus, this singularity is different from the well-known inverse square root singularity, oscillatory or not, respectively, at an interface crack tip in the so-called open and contact models of cracks at perfect interfaces (Mantič et al 2006).

\subsection{LEBIM as a model of an adhesive layer of a small thickness}

As discussed in Section 1, the LEBIM can be considered as a simple model of a thin linear elastic and brittle adhesive layer, whose material is relatively compliant 
in relation to the materials of adherents. It is assumed that stresses are constant across the thickness $h>0$ of the layer. The relationship of the properties of LEBIM to the mechanical and geometrical characteristics of the adhesive layer are discussed in this section.

Regarding the above linear elastic - (perfectly) brittle law in the normal direction represented in Fig. 1(a) and (c), once a portion of interface is broken, unrealistically large negative values for the normal relative displacement, $\delta_{n}<0$, are avoided by using the frictionless contact condition (12), see Fig. 1(c). The use of an elastic (frictionless) contact is based on the idea that the portions of the cracked layer remain on the adjacent surfaces, see Fig. 3 and also (Liechti 2002), for various types of crack paths in adhesive layers. Thus, when these surfaces enter in contact, it seems reasonable to assume that these portions of the layer could compress with the same stiffness, in the normal direction, as the layer had done before cracking.

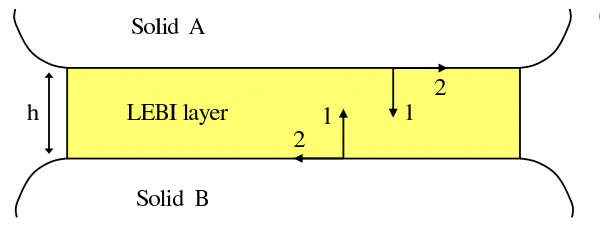

(a)

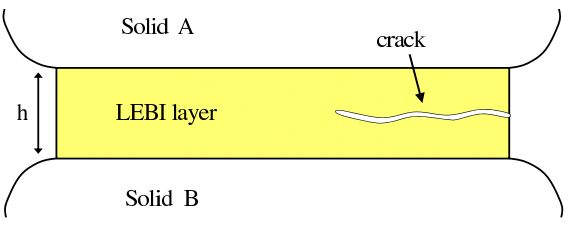

(b)

Fig. 3 LEBI layer (a) undamaged and (b) partially broken.

The stiffness parameters of the interface $k_{n}$ and $k_{t}$ can be related to the parameters of a linear elastic isotropic layer (Young's modulus $E_{\ell}$, Poisson's ratio $\nu_{\ell}$, shear modulus $\mu_{\ell}$, Lame's parameter $\lambda_{\ell}$ ) of a small thickness $h>0$ (Adams et al 1984; Kinloch 1987; Bank-Sills and Salganik 1994; Geymonat et al 1999; Benveniste and Miloh 2001; Hashin 2002; Távara et al 2011) by:

$$
k_{n}=\frac{2 \mu_{\ell}+\lambda_{\ell}}{h}=\frac{E_{\ell}^{\prime \prime}}{h}, \quad \text { and } \quad k_{t}=\frac{\mu_{\ell}}{h},
$$

where the apparent or constrained Young's modulus of the layer is defined as

$$
E_{\ell}^{\prime \prime}=\frac{E_{\ell}^{\prime}}{1-\nu_{\ell}^{\prime 2}}=\frac{E_{\ell}\left(1-\nu_{\ell}\right)}{\left(1+\nu_{\ell}\right)\left(1-2 \nu_{\ell}\right)}
$$

with $E_{\ell}^{\prime}=E_{\ell} /\left(1-\nu_{\ell}^{2}\right)$ and $\nu_{\ell}^{\prime}=\nu_{\ell} /\left(1-\nu_{\ell}\right)$. References to experiments verifying the above expression for $k_{n}$ can be found in (Kinloch 1987). From (14) and (15) the following expression of the ratio of $k_{t}$ and $k_{n}$ can be obtained:

$$
\xi=\frac{k_{t}}{k_{n}}=\frac{\mu_{\ell}}{2 \mu_{\ell}+\lambda_{\ell}}=\frac{1-\nu_{\ell}^{\prime}}{2}=\frac{1-2 \nu_{\ell}}{2\left(1-\nu_{\ell}\right)},
$$

leading to the following constraint for thin isotropic layers $0 \leq \xi=k_{t} / k_{n} \leq 0.5$. Moreover, a relation between $\bar{G}_{I c}, \bar{\sigma}_{c}, E_{\ell}^{\prime \prime}$ and $h$ is obtained from (6) and (14)

$$
\bar{G}_{I c}=\frac{\bar{\sigma}_{c}^{2} h}{2 E_{\ell}^{\prime \prime}} .
$$


This relationship corresponds to the fact that in the present formulation of LEBIM both the energetic and stress criteria are essentially equivalent and admit several interpretations which are discussed in the following.

If the strength in tension $\bar{\sigma}_{c}$ is measured experimentally, then for a given thickness $h$ and constrained Young's modulus $E_{\ell}^{\prime \prime}$ of the adhesive layer, the fracture energy $\bar{G}_{I c}$ can be obtained from (17) or viceversa. This approach has been adopted (explicitly or tacitly) by several researchers, e.g., a failure criterion for a thin adhesive layer, in terms of fracture energy, was proposed in (Erdogan 1997; Bennati et al 2009) and in terms of strength in (Caporale et al 2006).

If, however, both $\bar{\sigma}_{c}$ and $\bar{G}_{I c}$ of an adhesive, along with its elastic properties $E_{\ell}$ and $\nu_{\ell}$, are measured experimentally, then the present LEBIM works for the layer thickness $h$ verifying the condition $h=\bar{h}$, with $\bar{h}=\frac{2 \bar{G}_{I c} E_{\ell}^{\prime \prime}}{\bar{\sigma}_{c}^{2}}$ defining a threshold thickness from (17). To remove this restriction on the layer thickness, a coupled application of the stress and energy criteria, considered to be independently defined, was developed in the framework of Finite Fracture Mechanics (FFM) in (Cornetti et al 2012; Weißgraeber and Becker 2013; Muñoz-Reja et al 2014). For very thin adhesives with $h<\bar{h}$, these studies show that although the traction vector achieves its critical value, i.e. $t=t_{c}$, there is not sufficient energy stored in the layer to be released in order to break it, i.e. $G<G_{c}$, and a further increase to the applied load is required, possibly leading to crack advance by jumps of a finite size. According to the analysis in (Cornetti et al 2012), the present LEBIM can be viewed as the limit case for the adhesive thickness $h \rightarrow \bar{h}$. It is worth mentioning that the computational implementation of the present LEBIM formulation is extremely simple, in particular it is much more simple and computationally efficient than that of FFM+LEBIM, see (Muñoz-Reja et al 2014), where it is shown that for some problems the results obtained by both approaches can be quite close. On the other hand, for very thick adhesives with $h>\bar{h}$, it appears that when the traction vector achieves its critical value there is a surplus of elastic strain energy stored in the layer (there is more energy stored in the layer than there is necessary to break it), i.e. $G>G_{c}$, and an unstable dynamic crack propagation can be expected.

\subsection{Review of interface failure criteria in mixed mode}

The failure criterion for the LEBIM was introduced in a general way in Section 2.1. This criterion is viewed in greater detail here employing several phenomenological interface failure criteria in mixed mode found in the literature, some of them being widely accepted for perfect or cohesive interfaces.

Four energy or stress based parameterized families of interface failure criteria are described in the following list, including the defining equation and the corresponding dimensionless function $\hat{G}_{c}(\psi)$ in the present LEBIM formulation. In order also to cover interface failure due to shear under compression, a suitable generalization of the original versions of those criteria, which do not consider such a failure, is proposed here. Due to several dissipative mechanisms possibly taking place at the crack tip as asperity contact and plasticity (Evans et al 1990), and as has been evidenced in several test campaigns, e.g. (Wang and Suo 1990; Swadener et al 1999; Bank-Sills and Ashkenazi 2000), $G_{c}(\psi)$ in these criteria should increase 
with increasing $|\psi|$. This may require some conditions on their governing parameters. Convexity of the safe region of the tension-shear half-plane may also require some conditions on the governing parameters of these criteria.

- A widely accepted phenomenological law for the interface fracture energy as a function of mode mixity was proposed by Hutchinson and Suo (1992) (see a discussion in Thouless and Yang (2002)):

$$
\hat{G}_{c}(\psi)=1+\tan ^{2}(1-\lambda) \psi, \quad \text { with } \quad|\psi|<\bar{\psi}_{a}(\lambda), \quad 0 \leq \lambda \leq 1,
$$

where

$$
\bar{\psi}_{a}(\lambda)=\min \left\{\psi_{a}(\lambda), \pi\right\} \quad \text { and } \quad \psi_{a}(\lambda)=\frac{\pi}{2(1-\lambda)},
$$

and $\lambda$ is a fracture mode sensitivity parameter, adjusting the influence of mode II, which should be obtained experimentally. The limit $\lambda=1$ represents the ideally brittle interface with $\hat{G}_{c}(\psi)=1$, whereas for the limit $\lambda=0$ the crack advance depends only on the mode I component of ERR. A typical range $0.2 \leq \lambda \leq 0.3$ characterizes interfaces with moderately strong fracture mode dependence. The implicit equation of this law in the plane of normalized interface-tractions (8) can be obtained from (10).

- The so-called power law in terms of interface tractions covers some well-known stress based criteria such as Mohr-Coulomb $(p=1)$, quadratic $(p=2$, e.g. Brewer and Lagace (1988)), and maximum normal or shear traction $(p \rightarrow \infty)$ criterion, cf. García and Leguillon (2012). Its implicit equation in the plane of normalized interface-tractions (8) can naturally be extended, in the following two simple forms, to cover also compression regions as well as with $\hat{\sigma}_{c}<0$ :

$$
\begin{gathered}
\operatorname{sign}\left(\sigma_{c}\right)\left|\hat{\sigma}_{c}\right|^{p}+\mu^{-p}\left|\hat{\tau}_{c}\right|^{p}=1, \quad \text { or } \\
\left\langle\hat{\sigma}_{c}\right\rangle_{+}^{p}+\mu^{-p}\left|\hat{\tau}_{c}\right|^{p}=1, \\
\text { with } \quad p>0, \quad \mu=\frac{\bar{\tau}_{c}}{\bar{\sigma}_{c}}, \quad \bar{\sigma}_{c}, \bar{\tau}_{c}>0,
\end{gathered}
$$

Usually $p \geq 1$. The first extension to a compression region $\hat{\sigma}_{c}<0$ was proposed by García et al (2015), and coincides with the proposal made by Lemaitre and Desmorat (2005) which corresponds to $p=2$, while the second extension can be found in Harper et al (2012), again corresponding for $p=2$. The dimensionless fracture energy is defined by

$$
\hat{G}_{c}(\psi)= \begin{cases}\left(\cos ^{p} \psi+\mu^{-p} \xi^{p / 2}|\sin \psi|^{p}\right)^{-2 / p}, & |\psi| \leq \frac{\pi}{2}, \\ \left(-|\cot \psi|^{p}+\mu^{-p} \xi^{p / 2}\right)^{-2 / p}, & |\psi| \geq \frac{\pi}{2},\end{cases}
$$

in the first case (20a), whereas in the second case (20b) its definition for $|\psi| \geq \frac{\pi}{2}$ is different and is given by a constant value

$$
\hat{G}_{c}(\psi)=\mu^{2} \xi^{-1}, \quad|\psi| \geq \frac{\pi}{2} .
$$

The well-known power law in terms of ERRs is defined by the equation (Camanho et al 2003; Harper et al 2012)

$$
\hat{G}_{I c}^{q}+m^{-q} \hat{G}_{I I c}^{q}=1, \quad \text { with } \quad q>0, \quad m=\frac{\bar{G}_{I I c}}{\bar{G}_{I c}} .
$$


The particular case $q=1$ was suggested by Wang and Suo (1990) and used by Harper et al (2012). Pinho et al (2006) obtained $q=1.21$ by fitting some experimental data. It is easy to check that the energy based failure criterion (22) is equivalent to the above power law (in the present LEBIM), in terms of interface tractions (20), at least in the tension region $\left(|\psi| \leq \frac{\pi}{2}\right)$, taking $p=2 q$ and $m=\mu^{2} \xi^{-1}$. It can be confirmed that $\hat{G}_{c}(\psi)$ in (21a) is an increasing function for $|\psi| \leq \frac{\pi}{2}$, in agreement with the experiments, when and only when $p=2 q=2$, see Camanho et al (2003) for a related discussion. This observation makes a particularly relevant case for the stress based quadratic criterion and the equivalent energy based linear criterion among all power laws.

- The fracture energy dependence on mode mixity suggested by Benzeggagh and Kenane (1996) takes the following form in the present notation:

$$
\hat{G}_{c}(\psi)=\left\{\begin{array}{l}
1+(m-1) \sin ^{2 \eta} \psi,|\psi| \leq \frac{\pi}{2}, \\
m, \\
|\psi| \geq \frac{\pi}{2},
\end{array} \quad \text { with } \quad \eta>0, \quad m=\frac{\bar{G}_{I I c}}{\bar{G}_{I c}}\right.
$$

Ducept et al (1999) suggested the use of $\eta=1.5$, which was applied, e.g., by Bennati et al (2009), while Camanho et al (2003) obtained $\eta=2.28$ by fitting some experimental data and Goutianos and Sørensen (2012) used $\eta=2$. A simple form of the implicit equation in the plane of normalized interfacetractions can be obtained from (10),

$$
\begin{cases}\hat{\sigma}_{c}^{2}+\xi^{-1} \hat{\tau}_{c}^{2}=1+(m-1)\left(1+\xi \frac{\hat{\sigma}_{c}^{2}}{\hat{\tau}_{c}^{2}}\right)^{-\eta} & , \hat{\sigma}_{c} \geq 0 \\ \hat{\tau}_{c}^{2}=m \xi, & \hat{\sigma}_{c} \leq 0\end{cases}
$$

It is easy to check that the safe region in the tension-shear half-plane is not convex for some combinations of governing parameters $m$ and $\eta$. Moreover, in some cases even $\hat{\sigma}_{c}>1$ can be obtained for $\hat{\tau}_{c} \neq 0$, e.g., for $\eta=1$ and 2 , respectively, with $m>2$ and $m>5$.

- A simple linear variation of $\hat{G}_{I c}$ with $\psi$ was proposed, e.g., by Goutianos and Sørensen (2012), cf. Varna et al (1997),

$$
\hat{G}_{c}(\psi)=1+\frac{2(m-1)}{\pi}|\psi|, \quad \text { with } \quad m=\frac{\bar{G}_{I I c}}{\bar{G}_{I c}} .
$$

However, it is easy to show that its safe region in the tension-shear half-plane is non-convex for $m>1$, with $\hat{\sigma}_{c}>1$ for some $\hat{\tau}_{c} \neq 0$.

Other laws for $\hat{G}_{c}(\psi)$, which are not represented in the above list, can be obtained, e.g., by evaluating plastic dissipation in the bulk near the interface crack tip (Tvergaard 2001) or by considering a plastic interface slip (Panagiotopoulos et al 2013; Vodička et al 2014).

The phenomenological law proposed by Hutchinson and Suo (1992) is adopted in the present study for the analysis of a cylindrical inclusion under biaxial transverse loads and will be described in the following sections in more detail. In this case, the LEBIM is defined by only four independent parameters, e.g.: $\bar{G}_{I c}, \bar{\sigma}_{c}$ and dimensionless $\xi$ and $\lambda$. As discussed above, an extended range of validity for the original definition of $\hat{G}_{c}(\psi)$ is assumed here. The graphs for the interface failure curves parameterized by equations (9), on the plane of normalized interface 
stresses $\left(\sigma / \bar{\sigma}_{c}, \tau / \bar{\sigma}_{c}\right)$, considering $\xi=k_{t} / k_{n}=0.25$, are shown in Fig. 4, where only the upper half of these curves, for $\tau \geq 0$, is plotted. According to Fig. 4, an interface failure under compressions is possible but requires larger shear stresses, except for the case where $\lambda=1$. As a consequence, a closed crack with compressions in the neighbourhood of the crack tip, may propagate in the presence of sufficiently large shear stresses. Note that these curves have a continuous slope at $\sigma=0$, corresponding to $|\psi|=\left|\psi_{\sigma}\right|=\frac{\pi}{2}$.

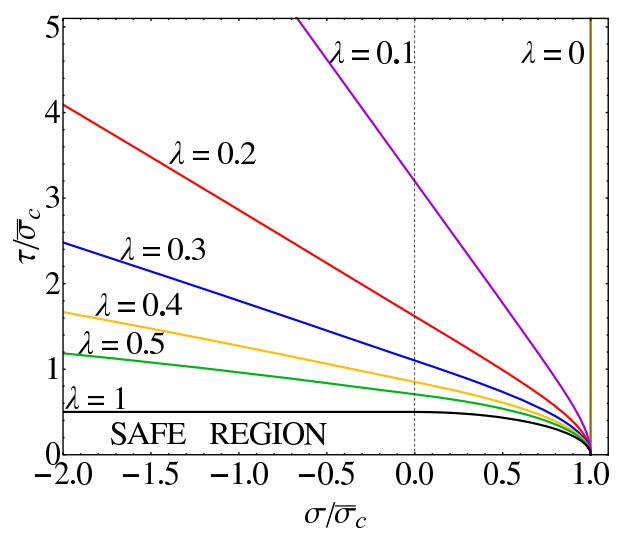

Fig. 4 Interface failure curves $(\hat{\sigma}(\psi), \hat{\tau}(\psi))$ on plane a $\left(\sigma / \bar{\sigma}_{c}, \tau / \bar{\sigma}_{c}\right)$ using Hutchinson and Suo (1992) law with different values of $\lambda$ and $\xi=k_{t} / k_{n}=0.25$.

It is easy to see from the expression $(18)$ that $\hat{G}_{c}(\psi)$ is unbounded for $0 \leq$ $\lambda \leq 0.5$ and $|\psi| \rightarrow \psi_{a}(\lambda)$. The angles $\pm \psi_{a}(\lambda)$ define, through the relation (3), the angles of the failure curve asymptotes for $0 \leq \lambda<0.5$. The failure curve for $\lambda=0.5$ has no horizontal asymptote, whereas the failure curves for $0.5<\lambda<1$ have horizontal asymptotes, and finally, the failure curve for $\lambda=1$ represents a constant $\tau_{c}(\psi)=\bar{\sigma}_{c} \sqrt{\xi}$ for $|\psi| \geq \frac{\pi}{2}$.

It is noteworthy that failure curves in Fig. 4 are similar to other interface failure curves like those presented by Lemaitre and Desmorat (2005) (See Fig. 7.5) and by Bialas and Mróz (2005a,b).

The failure (damage) for a portion of the interface layer is modeled as an abrupt decrease (jump down) of stresses in this zone of the layer. It is associated with a free separation or sliding of both interface surfaces, when a point on the failure curve (in $\left(\sigma / \bar{\sigma}_{c}, \tau / \bar{\sigma}_{c}\right)$ plane) is achieved in that portion of the layer. Actually, in view of Fig. 1, in the interface portion under compression only shear stresses jump down after its failure.

\section{The problem of a cylindrical inclusion under biaxial transverse loads}

The plane strain problem of a cylindrical inclusion (fibre) with a circular transverse section of radius $a>0$ embedded in an infinite matrix, initially without any debond along its interface, and subjected to remote transverse uniform stresses is considered. The materials of both the inclusion and the matrix are considered 
to be linear, elastic and isotropic. Let $(x, y)$ and $(r, \theta)$ be the cartesian and polar coordinates with the origin of coordinates in the center of the inclusion, assuming without any loss of generality that $(x, y)$ is the principal coordinate system of the remote stress state defined by the principal stresses $\sigma_{x}^{\infty} \geq \sigma_{y}^{\infty}$, see Fig. 5 .

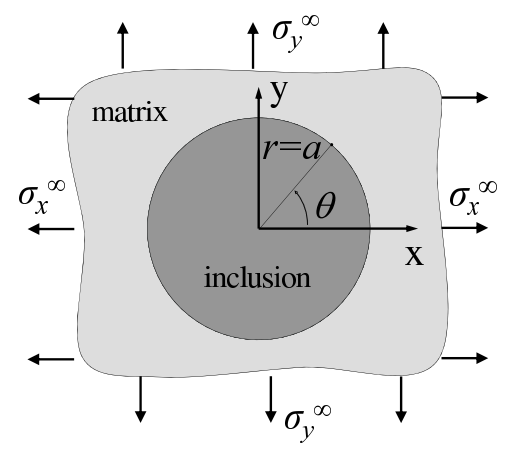

(a)

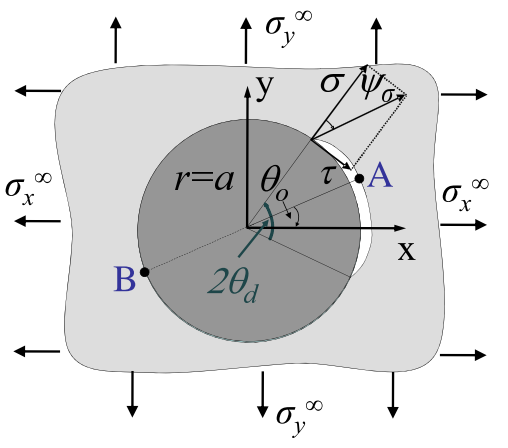

(b)

Fig. 5 Inclusion problem configuration under biaxial remote transverse tension (a) without and (b) with a partial debond.

Although the ratio of the principal stresses $\eta=\sigma_{y}^{\infty} / \sigma_{x}^{\infty}=\tan \phi^{\infty}$ is sometimes used to characterize the biaxiality of the remote stress state (Mantič and García 2012), in the present study, which covers also configurations where both remote principal stresses are compressive, the following general load-biaxiality parameter ${ }^{1}$ :

$$
\chi=\frac{\sigma_{x}^{\infty}+\sigma_{y}^{\infty}}{2 \max \left\{\left|\sigma_{x}^{\infty}\right|,\left|\sigma_{y}^{\infty}\right|\right\}}, \quad-1 \leq \chi \leq 1
$$

is more suitable. Denoting the Frobenius norm of the remote stress state by $\sigma_{F}^{\infty}=$ $\sqrt{\left(\sigma_{x}^{\infty}\right)^{2}+\left(\sigma_{y}^{\infty}\right)^{2}}$, we have $\sigma_{x}^{\infty}=\sigma_{F}^{\infty} \cos \phi^{\infty}$ and $\sigma_{y}^{\infty}=\sigma_{F}^{\infty} \sin \phi^{\infty}$.

Let the position where the interface crack onset occurs be defined by the polar angle $\theta_{o} \in\left\langle 0, \frac{\pi}{2}\right\rangle$. The semidebond angle is denoted as $\theta_{d}$. During the debond growth the angle $\theta_{o}$ may or may not be placed at the center of the debond.

According to Fig. 5(b) only one debond, initiated at a point $\mathrm{A}\left(r=a, \theta=\theta_{o}\right)$, is considered, although depending on the problem symmetry, two or four equivalent positions for debond onset may exist at the inclusion interface with $\theta= \pm \theta_{o}$, $\pm \theta_{o}+\pi$. Nevertheless, according to the experimental evidence only one side of the fibre-matrix interface is usually broken (Zhang et al 1997; Correa et al 2007). This will also be obtained by the present numerical model in Section 5, where the crack onset can occur at any of these two or four points, but once a crack has started at one of these points it will continue growing, preventing failure in the other symmetrically situated points.

1 It is easy to see that $\chi$ gives the position of the center of the normalized Mohr circumference and its characteristic values are $\chi=1$ (equibiaxial tension), $\chi=0.5$ (uniaxial tension), $\chi=0$ (equibiaxial tension-compression or pure shear stress), $\chi=-0.5$ (uniaxial compression) and $\chi=-1$ (equibiaxial compression). It is useful to realize that $\phi^{\infty}=\frac{\pi}{2}\left(\chi-\frac{1}{2}\right)$. 
A typical bi-material system among fibre reinforced composite materials is chosen for this study: $m$-epoxy matrix and $i$-glass fibre (inclusion), the elastic properties of matrix and inclusion being $E_{m}=2.79 \mathrm{GPa}, \nu_{m}=0.33, E_{i}=70.8$ GPa and $\nu_{i}=0.22$, respectively. The corresponding Dundurs bi-material parameters in plane strain are $\alpha=0.919$ and $\beta=0.229$, and the harmonic mean of the effective elasticity moduli is $E^{*}=6.01 \mathrm{GPa}$, see (París et al 2007; Mantič 2009; Mantič et al 2006; Soden et al 1998; Fiedler et al 2001) for their definitions.

The strength and fracture properties of the fibre-matrix interface, tensile strength $\bar{\sigma}_{c}=90 \mathrm{MPa}$ and fracture energy in mode I $\bar{G}_{I c}=2 \mathrm{Jm}^{-2}$, considered in the numerical procedure are in the range of values found in the literature (Zhang et al 1997; Varna et al 1997) and correspond to quite brittle behaviour (Mantič 2009; Mantič and García 2012), making the hypothesis for the LEBIM represent appropriately a real possible composite material behavior (Távara et al 2011). Then, $k_{n}$ is determined by (6).

A dimensionless structural parameter, referred to as the brittleness number, governing the brittle-to-tough transition in the fibre-matrix debond onset can be defined following (Mantič 2009; Távara et al 2011; Mantič and García 2012) as

$$
\gamma=\frac{1}{\bar{\sigma}_{c}} \sqrt{\frac{\bar{G}_{I c} E^{*}}{a}}
$$

In the present LEBIM formulation, $\gamma$ can also be expressed, in view of (6), as $\gamma=\sqrt{\frac{E^{*}}{2 k_{n} a}}$, showing that it is given by the ratio of the stiffness of the bimaterial $\left(E^{*}\right)$ and interface $\left(k_{n}\right)$ with the unique characteristic length of problem geometry (fibre diameter $2 a$ ). Small values of $\gamma$ (typically $\gamma \lesssim 1$ ) correspond to brittle configurations and large values of $\gamma$ (typically $\gamma \gtrsim 1$ ) to tough configurations. Noteworthy $\gamma$ is closely related to a similar dimensionless parameter $\delta$ defined by Lenci (2001) for a crack of size $2 a$ at a weak interface, verifying $\gamma \sim 1 / \sqrt{\delta}$.

In the following numerical study, some parametric analyses will be presented, all of them considering a default configuration with $\xi=0.25, \lambda=0.25$ and a circular inclusion radius $a=7.5 \mu \mathrm{m}$, leading to $\gamma=0.44$.

\section{Analytical and numerical procedures applied}

In section 4.1, firstly, the analytical solution for the above defined problem of a circular inclusion (fibre) under remote biaxial transverse loads, considering the inclusion-matrix interface as linear-elastic without any debond, is presented and discussed. Secondly, using this solution and the hypotheses for the LEBIM, an analytical procedure, able to evaluate a failure curve and the angle where debond onset takes place, is proposed. In section 4.2, the BEM model used to analyse debond onset and propagation at the interface in this problem is briefly described.

4.1 Analytical procedure applied to analyse the fibre-matrix debond onset

By using a closed-form expression of the Airy stress function deduced by Gao (1995) for an elastic, circular inclusion (fibre) embedded in an elastic, infinite matrix with an undamaged interface, the following expressions of interface tractions 
can be obtained, assuming uniform biaxial stresses, $\sigma_{x}^{\infty}$ and $\sigma_{y}^{\infty}$, at infinity:

$$
\begin{aligned}
& \sigma(r=a, \theta)=\frac{k_{n} a\left(1+\kappa_{m}\right)}{2 A C}\left\{\sigma_{x}^{\infty}(A+B C \cos (2 \theta))+\sigma_{y}^{\infty}\left(A+B C \cos \left(2\left(\theta+\frac{\pi}{2}\right)\right)\right)\right\}, \\
& \tau(r=a, \theta)=-\frac{k_{t} a\left(1+\kappa_{m}\right) D}{2 A}\left\{\sigma_{x}^{\infty} \sin (2 \theta)+\sigma_{y}^{\infty} \sin \left(2\left(\theta+\frac{\pi}{2}\right)\right)\right\},
\end{aligned}
$$

where

$$
\begin{gathered}
A=12 \mu_{m}^{2}+a^{2} k_{n} k_{t}\left(\kappa_{m}+t\right)\left(1+\kappa_{i} t\right)+a \mu_{m}\left(k_{n}+k_{t}\right)\left(1+3 \kappa_{m}+\left(3+\kappa_{i}\right) t\right), \\
B=6 \mu_{m}+a k_{t}\left(1+\kappa_{i} t\right), \\
C=4 \mu_{m}+a k_{n}\left(2+\left(\kappa_{i}-1\right) t\right), \\
D=6 \mu_{m}+a k_{n}\left(1+\kappa_{i} t\right),
\end{gathered}
$$

with $t=\mu_{m} / \mu_{i}$, and $\mu_{m}=E_{m} / 2\left(1+\nu_{m}\right)$ and $\kappa_{m}=3-4 \nu_{m}$, respectively being, the shear modulus and Kolosoff's constant of the matrix $(m)$, and analogously for the inclusion $(i)$. Equations (28) and (29) generalize expressions (27)-(31) introduced in Távara et al $(2011)^{2}$ for the uniaxial loading case $\left(\sigma_{y}^{\infty}=0\right)$.

Taking into account that the parameters $A, B, C$ and $D$ can be written in terms of $\gamma, \xi$ and of the elastic properties of matrix and inclusion (Távara et al 2011), and that $k_{n} a=E^{*} / 2 \gamma^{2}$, the interface tractions in (28) can be expressed in terms of the dimensionless functions $\hat{\sigma}$ and $\hat{\tau}$ as:

$$
\begin{aligned}
& \sigma(r=a, \theta)=\sigma_{F}^{\infty} \tilde{\sigma}\left(\theta ; \chi, \xi, \gamma ; E_{i} / E_{m}, \nu_{i}, \nu_{m}\right), \\
& \tau(r=a, \theta)=\sigma_{F}^{\infty} \tilde{\tau}\left(\theta ; \chi, \xi, \gamma ; E_{i} / E_{m}, \nu_{i}, \nu_{m}\right),
\end{aligned}
$$

where $\xi(16), \chi(26)$ and $\gamma(27)$ are governing dimensionless parameters.

Pseudocode for the proposed procedure for the evaluation of a failure curve in the plane of normalized remote stresses $\left(\sigma_{x}^{\infty} / \bar{\sigma}_{c}, \sigma_{y}^{\infty} / \bar{\sigma}_{c}\right)$, which uses the above analytical solution for interface tractions and assumes the hypotheses of the LEBIM, is introduced in Fig. 6. Additionally, this procedure evaluates the polar angle $\theta_{o}$ where the debond initiates. The procedure is self-explanatory, its detailed description being omitted for the sake of brevity.

The procedure presented in Fig. 6 predicts the critical biaxial load for each given load biaxiality parameter $\chi$ leading to the failure of the first interface point. However, it is not clear if this initial infinitesimal debond will grow further, in an unstable way, under the same critical load or if an additional increase to this load will be required to keep the infinitesimal debond growing. This question will be answered applying the numerical procedure briefly described in the next section.

4.2 The numerical procedure used to analyse the fibre-matrix debond onset and propagation

The present non-linear problem of the crack onset and propagation along the fibrematrix interface governed by the LEBIM can be solved either by means of FEM

\footnotetext{
2 There are several misprints in Eqs. (27)-(31) in Távara et al (2011) corrected herein
} 


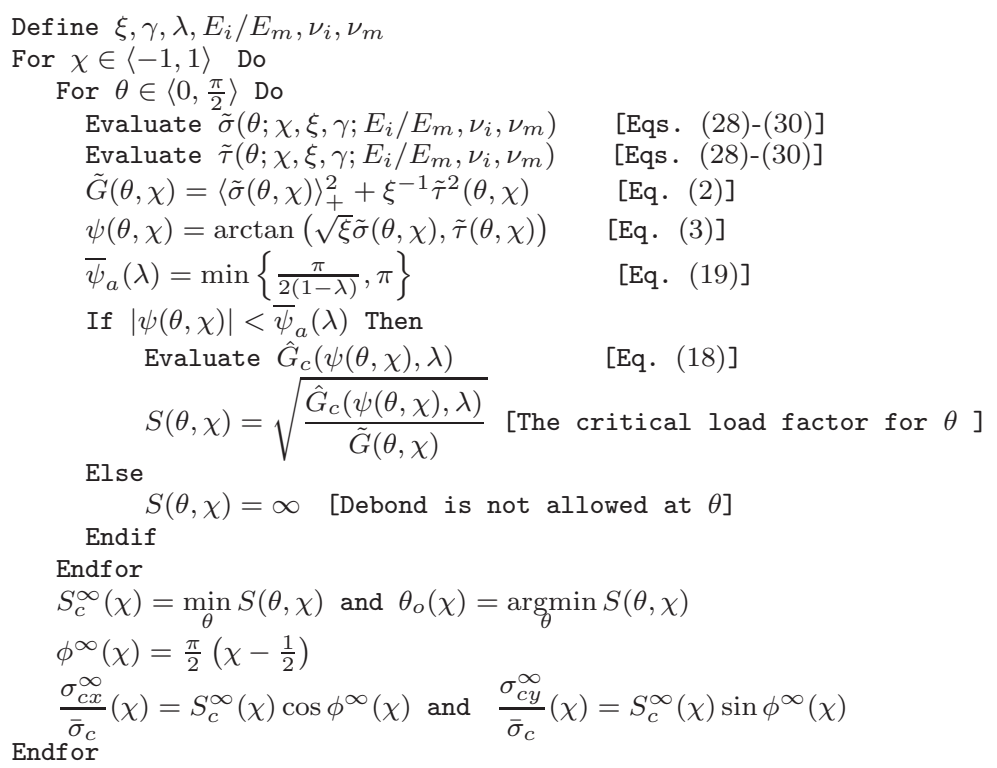

Fig. 6 Procedure for the evaluation of the normalized failure curve $\left(\sigma_{c x}^{\infty}(\chi) / \bar{\sigma}_{c}, \sigma_{c y}^{\infty}(\chi) / \bar{\sigma}_{c}\right)$ and angle $\theta_{o}(\chi)$ where the debond initiates, for a circular inclusion subjected to remote biaxial loads.

or BEM. The BEM, which is very suitable for solving these kinds of problems where all nonlinearities are placed on the boundaries of the subdomains, is employed in the present work. Implementation details of the collocational 2D BEM code employed and an overall description of the solution algorithm can be found in (Távara et al 2011, 2010; Távara 2010; Graciani et al 2005). This algorithm uses an incremental formulation and a very efficient solution procedure, usually referred to as sequentially linear (elastic) analysis, appropriate for the present nonlinear problem (Távara et al 2011, 2010). Pseudocode of this procedure in the case of proportional loading (boundary conditions), defined by a nominal load value multiplied by a dimensionless load factor $F \geq 0$, is presented in Fig. 7 .

The inclusion is initially considered as bonded to the matrix along its perimeter by means of a continuous distribution of springs governed by the LEBIM. The debond onset and propagation is modeled by progressively breaking springs placed between boundary element nodes positioned at both sides of the interface. Therefore, the numerical procedure used is driven by the interface crack length and is able to analyse both snap-through and snap-back instabilities of a crack growth.

The present BEM model represents a circular inclusion with a radius $a=7.5$ $\mu \mathrm{m}$ inside a relatively large square matrix with side $2 \ell=1 \mathrm{~mm}$. The BEM mesh has 1472 continuous linear boundary elements: two uniform meshes of 720 elements discretizing both sides of the fibre-matrix interface (therefore, the polar angle of each element is $0.5^{\circ}$ ) and 32 elements for the external boundary of the matrix, where the remote stresses $\sigma_{x}^{\infty}$ and $\sigma_{y}^{\infty}$ are applied. Rigid body motions are removed by Method F2 introduced in Blázquez et al (1996), see also Graciani et al (2005). 


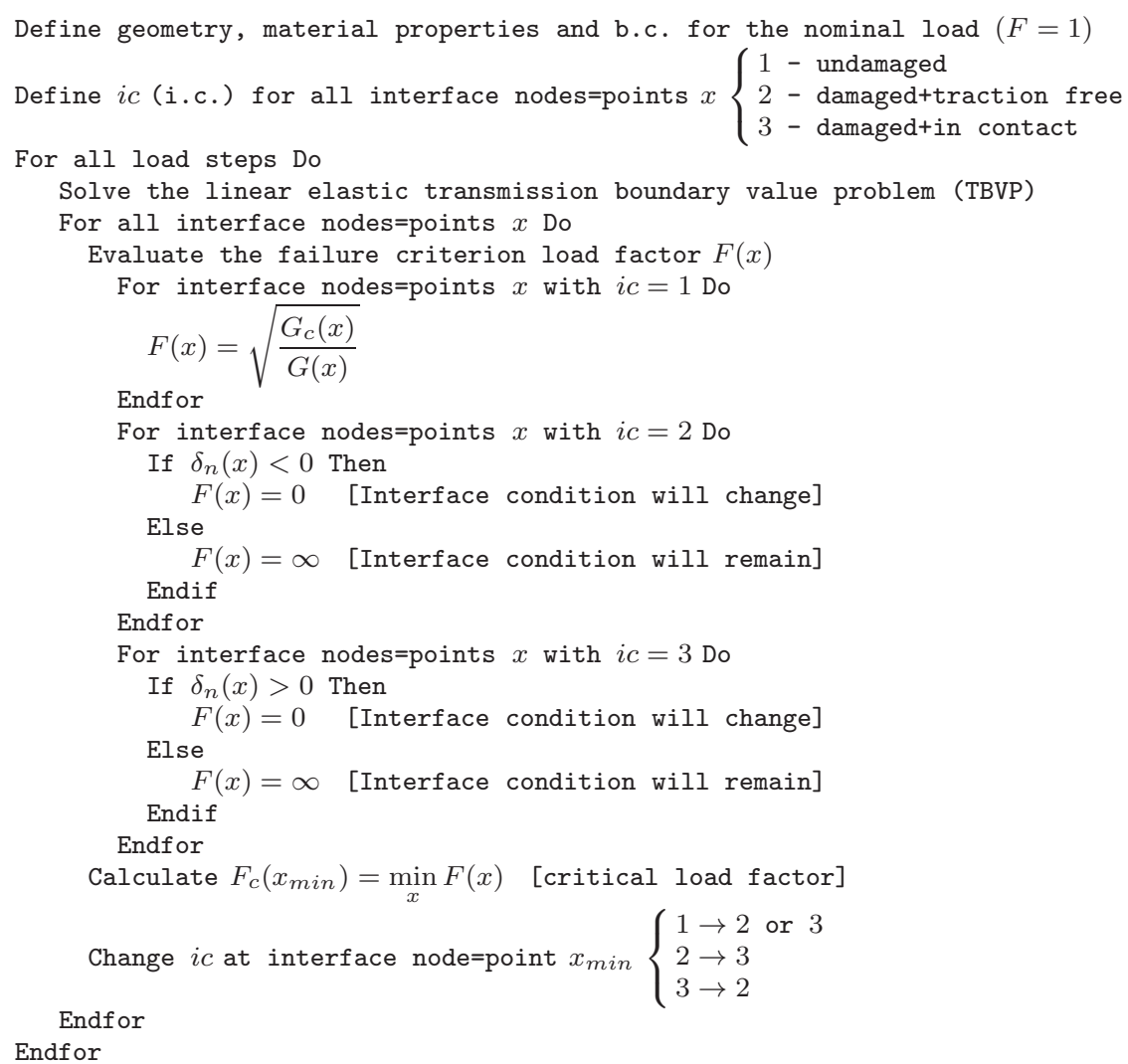

Fig. 7 Procedure of the sequentially linear (elastic) analysis applied to a problem including an interface governed by the LEBIM, where b.c.=boundary conditions and i.c.=interface condition.

\section{Results for the fibre-matrix debond onset and propagation}

The aim of this section is to study the influence of the governing parameters $\xi(16), \lambda(18), \chi(26)$ and $\gamma(27)$ of the present model on the debond onset and propagation in the case of the glass-fibre and epoxy-matrix composite (Section 3). Firstly, the debond onset is studied focusing on the angle of debond onset as a function of the remote stress biaxiality (Section 5.1) and on the failure curves in the plane of normalized remote stresses (Section 5.2). Secondly, debond growth is studied by evaluating load-debond opening curves and load-debond length curves (Section 5.3). Finally, an instability analysis of the debond onset and growth is introduced (Section 5.4).

Both of the analytical and numerical procedures developed are applied wherever feasible, and their results compared, which allows us to mutually verify the correctness of the implementation of these procedures. The analytical procedure is highly suitable for some of the parametric studies presented. However, its range of application is limited to the debond onset characterization in the present problem for a single fibre embedded in an infinite matrix. The scope of the numerical procedure developed is much wider and will allow us to solve complex, realistic 
problems to do with concurrent debond onset and propagation in dense fibre packing; this will include random distribution of many fibres in the future, cf. (Távara 2010; Távara et al 2013).

\subsection{Position of the crack onset}

The position where the crack onset occurs, defined by the angle $\theta_{o}$, Fig. 5 , is studied by means of the analytic procedure introduced in Section 4.1. In order to develop an efficient parametric study only the analytic procedure was used for this part, nevertheless it could also be studied by means of the numerical procedure. Graphs of $\theta_{o}(\chi ; \xi, \lambda, \gamma)$ in Fig. 8 show the influence of different governing parameters on this angle. For a clearer view, only the range $-0.55 \leq \chi \leq 0.5$ is shown. In the range $0.5 \leq \chi<1, \theta_{o}=0$ in all the cases studied. Obviously, for $\chi=1$ (remote equibiaxial tension), all interface points are equivalent and $\theta_{o}$ is undetermined. On the contrary for $-1 \leq \chi \leq-0.55$ a limit is reached, for each value of $\chi$, where no debond onset is possible due to the asymptotic behavior of the failure curves in Fig. 2 this demonstrates that an interface can break only if $|\psi|<\bar{\psi}_{a}\left(\bar{\psi}_{a}\right.$ defined in Fig. 6).

According to these graphs of $\theta_{o}(\chi)$, a bifurcation takes place at a particular value of $\chi$, referred to as bifurcation value $\chi_{b}(\xi, \lambda, \gamma)$. Then, for $\chi<\chi_{b},\left|\theta_{o}(\chi)\right|>0$ and for $\chi_{b} \leq \chi<1$ the first interface point breaks at $\theta_{o}=0$ in pure fracture mode I. This behaviour could be expected for tension dominated remote loads roughly characterized by $\chi>0$, taking into account the distribution of interface tractions (28) and the failure criterion in Fig. 4. Nevertheless, as will be seen, there are some exceptions observed for very brittle configurations and for fracture energies independent of the mode mixity. For $\chi<\chi_{b}$, a kind of bifurcation is observed due to a sudden variation of $\theta_{o}$ for $\chi$ below, and close, to $\chi_{b}$. In this case, the interface breaks in a mixed mode.

The influence of $\xi$ on $\theta_{o}$ is depicted in Fig. 8(a), showing that with an increasing value of $\xi$ the bifurcation value $\chi_{b}$ slightly increases as well. Nevertheless, it seems that when $\chi<-0.5$ decreases, the dependence of $\theta_{o}$ on $\xi$ becomes weaker.

Fig. 8(b) presents the influence of $\lambda$ on $\theta_{o}$, showing that with an increasing value of $\lambda$ the bifurcation value $\chi_{b}$ increases as well. Thus, for large values of $\lambda$ a non-symmetric debond initiation is predicted for biaxial tension-compression loading even if the tension is a little larger than the compression. For $\chi<\chi_{b}$ the value of $\theta_{o}$ increases with an increasing value of $\lambda$, which could be expected, as the interface failure criterion becomes more sensitive to the interface shear traction value according to Fig. 4.

From Fig. 8(c), showing the influence of $\gamma$ on $\theta_{o}$, it can be observed that for high values of $\gamma$ (tough configurations) no bifurcation takes place and $\theta_{o}=0$, predicting the debond onset in mode $\mathrm{I}$, for the considered values of $\chi,-0.55 \leq \chi<1$. However, for low values of $\gamma$ (brittle configurations) a non-symmetric debond is predicted for biaxial tension-compression loading even for relatively small values of the secondary compression load.

Although not shown in Fig. 8, for a low value of $\gamma$ and a high value of $\lambda$, e.g. $\gamma=0.1$ and $\lambda=0.5$, a non-symmetric debond initiation would be predicted even for the uniaxial tension, see Fig. $9(\mathrm{~d}-\mathrm{f})$. This somewhat surprising behaviour can be explained by the observation that the ratio of the maximum values of $\tau$ and 


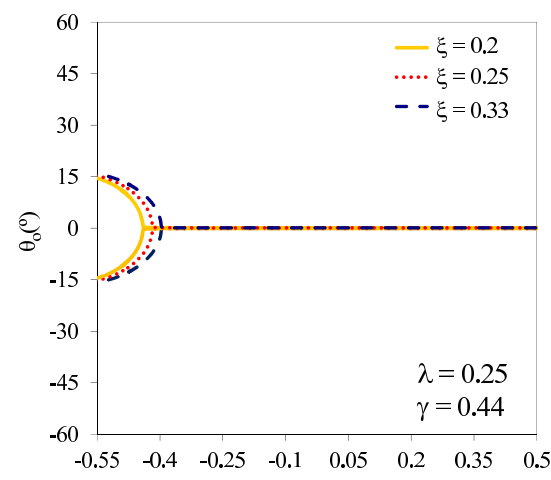

(a)

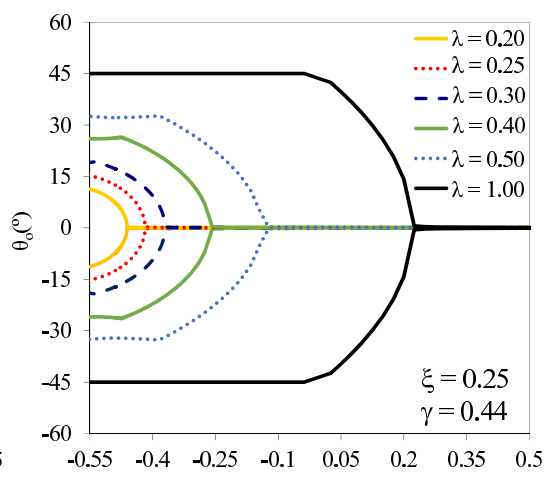

(b)

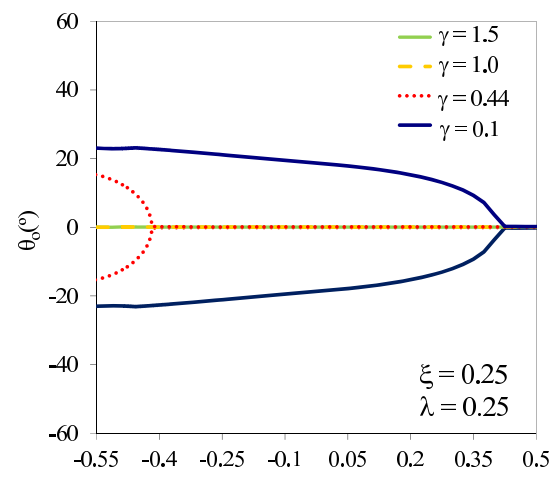

(c)

Fig. 8 Analytical results. Graphs of the crack onset angle $\theta_{o}$ as a function of the load biaxiality parameter $\chi$ for several values of the material and structural parameters (a) $\xi$, (b) $\lambda$ and (c) $\gamma$.

$\sigma$ in (28) is increasing for decreasing $\gamma$ (and/or decreasing $\chi$ ) making the debond onset easier in mixed mode. It is worthy of note that similar behaviour for the uniaxial tension has also been observed in predictions by other models such as CZM and FFM in García et al (2014).

Fig. 9 shows the deformed shape for uniaxial tension at different stages for two cases obtained numerically by means of BEM. It can be seen that for the first case with $\xi=0.25, \lambda=0.25$ and $\gamma=0.44$ the interface crack growth is symmetrical with respect to the applied load. However, in the second case with $\xi=0.25, \lambda=0.5$ and $\gamma=0.1$ an initially non-symmetrical growth is obtained, due to the position where the crack onset appears $\theta_{o} \neq 0$. Notice that although the onset may initially not be symmetric, the crack becomes symmetric after a certain number of steps, leading to a further symmetrical growth, see Fig. 9(f). 


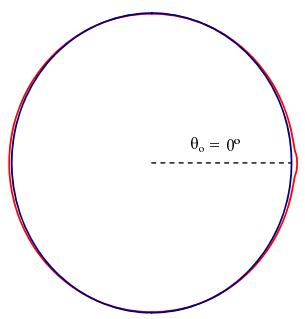

(a)

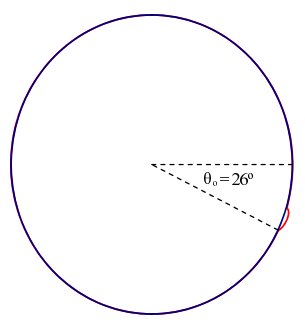

(d)

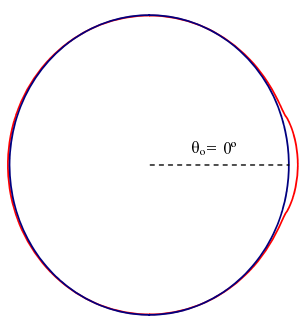

(b)

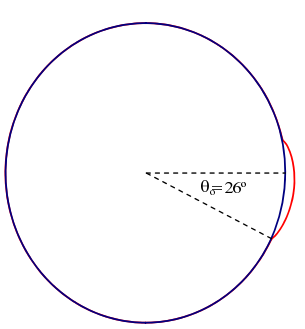

(e)

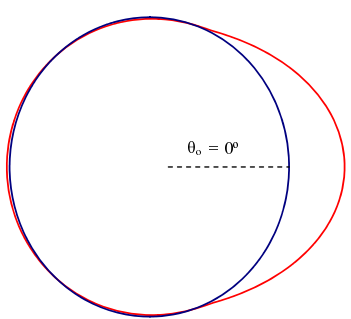

(c)

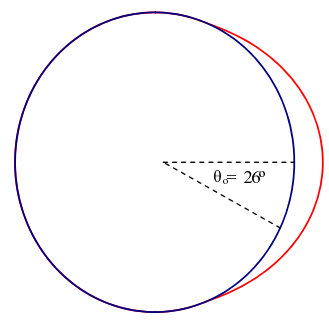

(f)

Fig. 9 Deformed shape for $\xi=0.25, \lambda=0.25, \gamma=0.44$ and uniaxial tension with $\chi=0.5>\chi_{b}$, obtained when (a) $2 \theta_{d}=10^{\circ}$, (b) $2 \theta_{d}=40^{\circ}$ and (c) $2 \theta_{d}=145^{\circ}$, displacements multiplied by a factor of 5. Deformed shape for $\xi=0.25, \lambda=0.5, \gamma=0.1$ and uniaxial tension with $\chi=0.5<\chi_{b}$, obtained when (d) $2 \theta_{d}=10^{\circ}$, (e) $2 \theta_{d}=40^{\circ}$ and (f) $2 \theta_{d}=145^{\circ}$, displacements multiplied by a factor of 5 .

\subsection{Failure curves}

Fig. 10 presents failure curves parameterized by the load biaxiality parameter $\chi$ and representing the normalized critical remote stresses which lead to the breakage of the first point (spring) of an initially undamaged inclusion-matrix interface. Analytical and numerical results are represented by continuous lines and markers, respectively. These graphs show the influence of the material ( $\xi$ and $\lambda$ ) and structural $(\gamma)$ dimensionless parameters of the problem on the failure curve shape and location. As can be observed from Fig. 10, an excellent agreement is achieved between the analytical and numerical procedures for several tension dominated biaxial loads (filled markers with $\xi=0.25, \lambda=0.25$ and $\gamma=0.44$ ) and a uniaxial compression load (empty marker with $\xi=0.25, \lambda=0.3$ and $\gamma=0.44$ ).

Regarding the influence of the load biaxiality parameter $\chi$, it is easy to observe in all the graphs in Fig. 10 that, considering $\sigma_{x}^{\infty} \geq \sigma_{y}^{\infty}$ (when looking at the rightbottom branch of failure curves), for decreasing values of $\chi$ the critical remote stress $\sigma_{c x}^{\infty}$ decreases quite significantly. In particular, a relevant compression $\sigma_{y}^{\infty}<$ 0 makes a debond onset appear more easily, $\sigma_{c x}^{\infty}$ being significantly smaller than in the case of a biaxial tension $(\chi>0.5)$ with $\sigma_{y}^{\infty}>0$, or even the uniaxial tension $(\chi=0.5)$ with $\sigma_{y}^{\infty}=0$. These observations coincide qualitatively with experiments by (París et al 2003). 


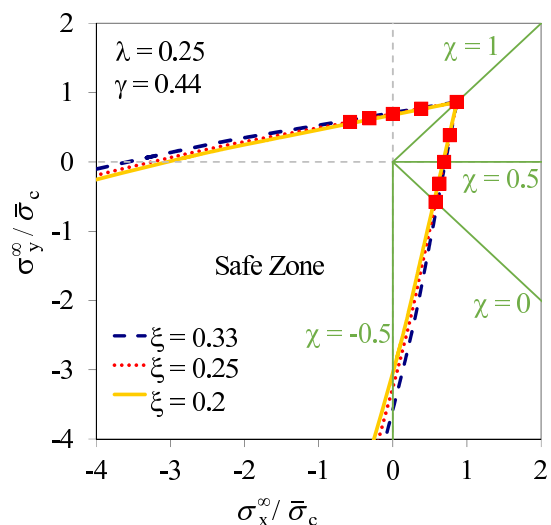

(a)

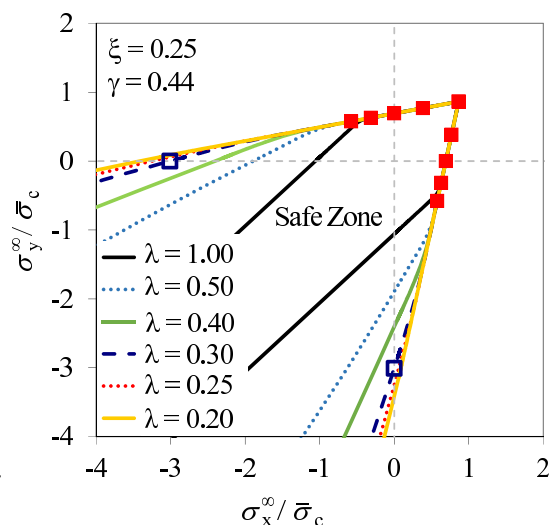

(b)

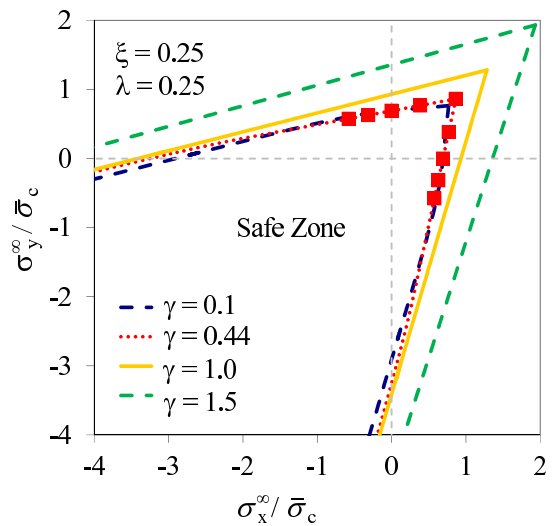

(c)

Fig. 10 Analytical (curves) and numerical (markers) results. Normalized failure curves of a circular inclusion under biaxial transversal loads for several values of the material and structural parameters (a) $\xi$, (b) $\lambda$ and (c) $\gamma$.

The rather weak influence of the ratio of the interface stiffness $\xi$ on the fibrematrix failure curve can be observed in Fig. 10(a) obtained by varying $\xi(\xi=0.20$, 0.25 and 0.33 ) and keeping constant the fracture mode-sensitivity parameter $\lambda=$ 0.25 and the brittleness number $\gamma=0.44$. For lower values of $\xi$ the critical loads are only slightly lower, this influence being mostly visible for $\chi<0$, and in particular for the case of the uniaxial compression $(\chi=-0.5)$.

The influence of the fracture mode-sensitivity parameter $\lambda$ on the failure curve is studied in Fig. 10 (b), by varying $\lambda(\lambda=0.2,0.25,0.3,0.4,0.5$ and, for completeness, also 1.0) and keeping constant both $\xi=0.25$ and $\gamma=0.44$. Considering, for example, $\lambda \leq 0.5$, there is no influence of $\lambda$ on the failure curve for $-0.12 \lesssim \chi \leq 1$, because in this range the crack onset occurs at $\theta_{o}=0$, see Fig. $8(\mathrm{~b})$, which, in view of the symmetry of the stress solution (28), means that shear tractions vanish there, and consequently the interface breaks in mode I at this point. Nevertheless, for larger values of compressions $\sigma_{y}^{\infty}$, i.e. $\chi \lesssim-0.12$, the crack onset changes its position given by $\theta_{o}>0$, see Fig. 8(b), the interface breaking in a mixed mode there. This leads to a strong influence of $\lambda$ on the shape of failure curves for this 
range of $\chi$. The critical loads are significantly lower for larger values of $\lambda$, because the interface strength strongly decreases with increasing $\lambda$ according to (9) with (18) and Fig. 4.

The influence of the brittleness number $\gamma$ on the failure curve is shown in Fig. 10(c), by varying $\gamma(\gamma=0.1,0.44,1$ and 1.5$)$ and keeping constant $\xi=0.25$ and $\lambda=0.25$. While the variations of failure curves for small values of $\gamma$ (brittle configurations) predicting small critical loads are hardly visible, a reasonably strong influence for $\gamma$ on the position of failure curves is observed for larger values of $\gamma$ (tough configurations) predicting large critical loads. Notice that, in view of the dependence of $\gamma$ on the inclusion radius $a(27)$, the variations of the failure curves with $\gamma$ represent in fact a size effect of $a$ on the crack onset, cf. (Mantič 2009; Távara et al 2011; Mantič and García 2012; Carpinteri et al 2005; García et al 2014).

Recall that the present formulation of the LEBIM, see Fig. 4, enables also crack onset under compressions as well in the presence of large shear tractions at the initiation point to be studied. This capability allows us to model crack onset, and later on growth, even in the case of remote compressions applied in both directions, i.e. for $\chi<-0.5$.

As can be observed in Fig. 10 there are some large differences between the failure curves, one of the reasons for these differences being the variations of the crack onset position given by the angle $\theta_{o}$, a question studied in Section 5.1.

\subsection{Effect of the load biaxiality on the fibre-matrix debond onset and growth}

The effect of the load biaxiality on the debond onset and growth is studied by the numerical procedure presented in Section 4.2. It will be shown that the failure curves presented in Fig. 10, referring to the breakage of the first interface point, actually represent the initiation of an unstable crack growth along the inclusionmatrix interface. The default values $\xi=0.25, \lambda=0.25$ and $\gamma=0.44$ are chosen for the following numerical study.

In Fig. 11 and Table 1 the numerical results obtained for different values of the load biaxiality parameter $\chi=0,0.25,0.5,0.75$ and 1 are presented. Recall that $\chi=0.5$ corresponds to the case of uniaxial tension in the $x$-direction $\left(\sigma_{y}^{\infty}=0\right)$.

In Fig. 11(a), the normalized remote stress $\sigma_{x}^{\infty} / \bar{\sigma}_{c}$ is plotted as a function of the normal relative displacement (opening) $\delta_{n}$ evaluated at the point $\mathrm{A}\left(a, \theta_{o}=0\right)$, sometimes referred to as Crack Mouth Opening Displacement (CMOD), point A indicated in Fig. 5(b). The (minimum) remote stress value that is needed to initiate crack growth (in simple terms, the remote stress that is needed to break the first spring in the present discrete model of the interface) is called critical stress, $\sigma_{c x}^{\infty}$, and corresponds to the local maximum of each curve shown in Fig. 11(a). It can also be observed in Fig. 11(a) that after reaching the critical stress, $\sigma_{c x}^{\infty}$, the crack growth becomes unstable, requiring smaller values of the remote tension to cause further crack growth. Thus, an instability phenomenon called snap-through is predicted under load control, see Section 5.4.

The variations of the local maxima values in Fig. 11(a) confirm the conclusion observed previously in Fig. 10 that the critical stress $\sigma_{c x}^{\infty}$ decreases with decreasing $\chi$, see also Table 1 . 


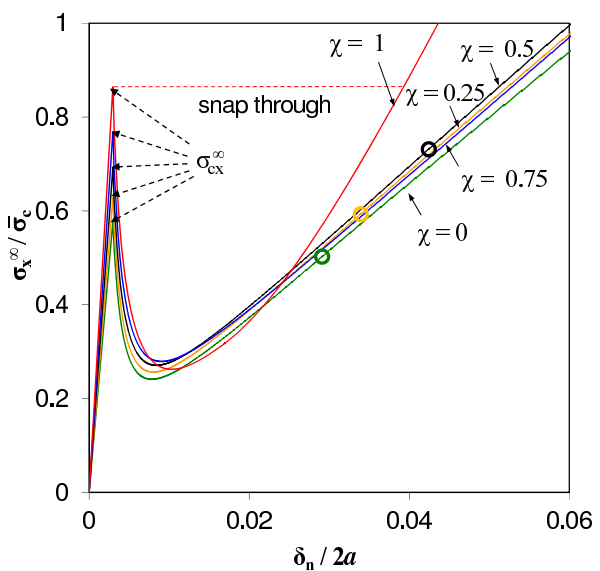

(a)

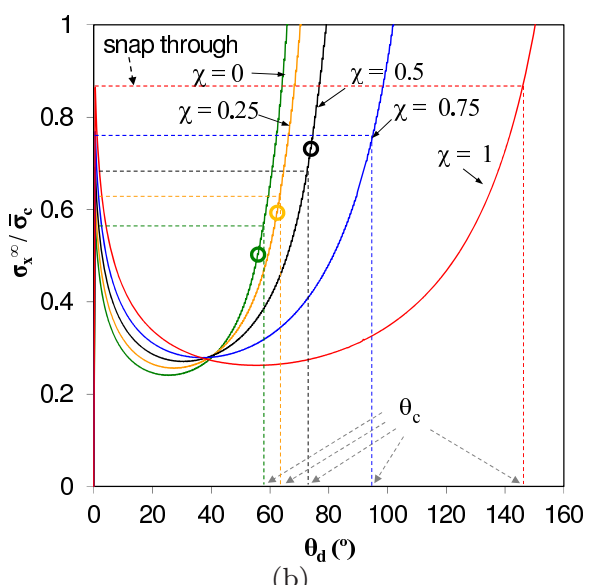

(b)

Fig. 11 Numerical results. (a) The normalized applied stress with respect to the normal relative displacements $\delta_{n}$ at point A, see Fig. 5, and (b) The normalized applied stress with respect to the semidebond angle $\theta_{d}$, for different biaxial loads combinations, with $\xi=0.25$, $\lambda=0.25$ and $\gamma=0.44$. The configurations for which contact between the crack faces is first detected are indicated by circles.

In Fig. 11(b), the normalized remote stress $\sigma_{x}^{\infty} / \bar{\sigma}_{c}$ is plotted versus the semidebond angle $\theta_{d}$ defined in Fig. $5(\mathrm{~b})$. An estimation of the critical semidebond angle $\theta_{c}$ defined as the semidebond angle $\theta_{d}$ reached at the end of the initial unstable crack growth, keeping the remote stress $\sigma_{c x}^{\infty}$ constant, is also shown in this figure. In general, $\theta_{c}$ increases with increasing $\chi$ in the range studied, see also Table 1 . When $\chi \gtrsim 0.75$, i.e. when significant remote tensions are applied in both axes, $\theta_{c}>\frac{\pi}{2}$. Thus, an unstable debond growth is predicted along a very large portion of the fibre-matrix interface.

Additionally, the values of the semidebond angle $\theta_{f}$ for which the contact zone between the crack faces is first detected in the numerical solution are included in Table 1, and the corresponding points on the curves in Fig. 11 are indicated by circles as well. It can be observed that the presence of friction would affect only in some cases $(\chi=0$ and 0.25$)$ the very last part of the unstable crack growth and consequently would not essentially affect the predicted values of $\theta_{c}$.

Table 1 The normalized critical stress for crack onset $\sigma_{c x}^{\infty}$ and critical semidebond angle $\theta_{c}$, for different values of $\chi$, with $\xi=0.25, \lambda=0.25$ and $\gamma=0.44$.

\begin{tabular}{|c|c|c|c|c|c|}
\cline { 2 - 6 } \multicolumn{1}{c|}{} & \multicolumn{5}{c|}{$\chi$} \\
\cline { 2 - 6 } \multicolumn{1}{c|}{} & 0 & 0.25 & 0.5 & 0.75 & 1 \\
\hline$\sigma_{c x}^{\infty} / \bar{\sigma}_{c}$ & 0.573 & 0.629 & 0.692 & 0.769 & 0.864 \\
$\theta_{c}\left({ }^{\circ}\right)$ & 58.25 & 63.25 & 72.75 & 95.25 & 146.0 \\
$\theta_{f}\left({ }^{\circ}\right)$ & 56 & 62.5 & 74 & 104 & $>166.25$ \\
\hline
\end{tabular}

Actually, the prediction of an unstable crack growth up to the critical semiangle $\theta_{c}$ is the key result obtained by the numerical solution of the present problem, 
as the values for $\sigma_{c x}^{\infty}$ and $\theta_{o}$ can also be obtained by the analytical procedure presented.

5.4 Instability analysis of the fibre-matrix debond onset and growth

In the following section, the instability behaviour (snap-through) observed in Fig. 11 will be further analysed to clarify its character under external load or displacement control. Only the case of uniaxial tension $(\chi=0.5)$, with the default values of $\xi, \lambda$ and $\gamma$, is considered hereinafter for the sake of brevity, the results will be similar for other values of the governing dimensionless parameters. Fig. 12(a) shows the normalized, applied, remote stress $\sigma_{x}^{\infty} / \bar{\sigma}_{c}$ versus the averaged longitudinal strain $\varepsilon$ along the segments between two pairs of points of the matrix placed on the $x$-axis and symmetrically situated with respect to the origin, $A B$ and $P Q$. The coordinates of the end points of $A B$ are $(x= \pm a, y=0)$ and of $P Q$ $(x= \pm \ell, y=0)$, where $a$ is the fibre radius and $\ell$ the half-length of the matrix square cell side, $\ell / a=66.7$ in the present study, as defined in Section 4.2. $\varepsilon^{e}$ represents the averaged longitudinal strain for a purely linear elastic fibre-matrix interface with no debond, while $\varepsilon^{d}$ is the additional averaged longitudinal strain due to debond $\left(\varepsilon^{d}=\varepsilon-\varepsilon^{e}\right)$. For a similar additive decomposition of relative displacements, see (Bažant and Cedolin 1991) (Ch. 12 therein).

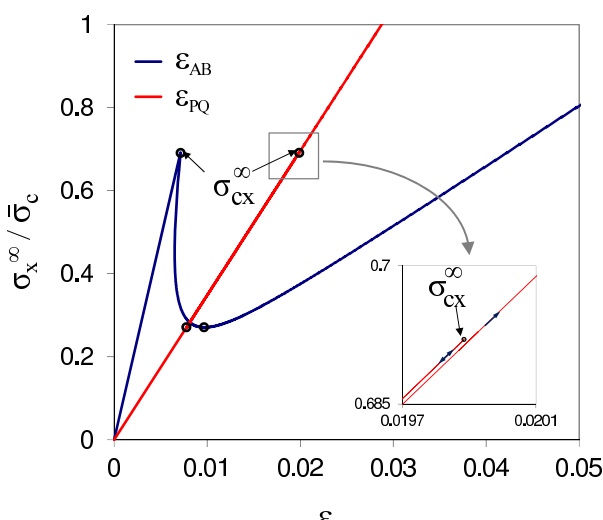

(a)

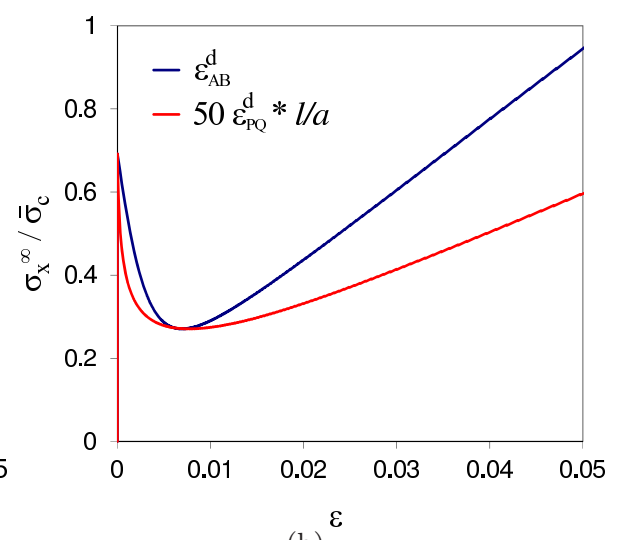

(b)

Fig. 12 Numerical results. The normalized applied stress $\sigma_{x}^{\infty}$ with respect to (a) the averaged longitudinal strains, $\varepsilon_{A B}=\varepsilon_{A B}^{e}+\varepsilon_{A B}^{d}$ and $\varepsilon_{P Q}=\varepsilon_{P Q}^{e}+\varepsilon_{P Q}^{d}$, and (b) the additional averaged longitudinal strain due to debond, $\varepsilon_{A B}^{d}$ and the scaled one $50 \frac{\ell}{a} \varepsilon_{P Q}^{d}$, with $\chi=0.5, \xi=0.25$, $\lambda=0.25$ and $\gamma=0.44$.

The diagrams $\sigma_{x}^{\infty}-\varepsilon$ in both cases (considering segments $A B$ and $P Q$ ) exhibit cusp snapback instability (Carpinteri 1989) after the peak point (bifurcation point) where the debond onset occurs. Actually, this kind of instability also appears for strains averaged along all intermediate segments between $A B$ and $P Q$. While the snapback instability is easily observable in the curve $\sigma_{x}^{\infty}-\varepsilon_{A B}$ in Fig. 12(a), this instability is not visible to the naked-eye in the curve $\sigma_{x}^{\infty}-\varepsilon_{P Q}$, as the curve's 
branches before and after the peak point are extremely close to each other, visually coinciding in the graph because the matrix cell is very large relative to the fibre. As the effect of the debond onset and growth at the fibre-matrix interface is hardly visible in this graph, a zoomed view of this curve with its cusp is also included in Fig. 12(a) to show this instability behaviour. Obviously the values of $\sigma_{x}^{\infty} / \bar{\sigma}_{c}$ at the local maxima (peak point) and minima in both curves coincide (values 0.692 and 0.2704 , respectively) as indicated in the graphs of the curve. It means that after the debond onset, we may decrease the applied load significantly, up to $39 \%$ of the critical load at its peak, keeping a continuous propagation of the debond.

Moreover, to better understand the post-peak behaviour, diagrams $\sigma_{x}^{\infty}-\varepsilon^{d}$ are plotted in Fig. 12(b). The value of $\varepsilon_{P Q}^{d}$, which strongly depends on the cell size $\ell$ (as a consequence of the Saint-Venant and superposition principles), is scaled by an arbitrary factor $50 \frac{\ell}{a}$ resulting in a value very similar to that of $\varepsilon_{A B}^{d}$. The initial very steep negative slope of these diagrams indicates that, according to the present LEBIM of the fibre-matrix interface, the debond onset and growth exhibits cusp snapback instability typical of a brittle structural behaviour. This observation is quite different from the smooth snapback instability observed in some cases in (Carpinteri et al 2005; García et al 2014) using a CZM for the fibre-matrix interface.

Summarizing the above analysis, the curve $\sigma_{x}^{\infty}-\varepsilon_{P Q}$ shows that under both load and displacement control at the outer boundaries of the matrix cell a sudden and large breakage of the fibre-matrix interface is predicted by the present model. Notice that after the debond onset, the growth could develop, at least hypothetically, in a stable manner under CMOD control, according to Fig. 11(a).

\section{Concluding remarks}

A new generalized formulation for a linear elastic - (perfectly) brittle interface model (LEBIM) was developed. This model covers mixed-mode interface-fracture under tensions as well as compressions in the presence of sufficiently large shear stresses, and considers frictionless linear elastic contact after interface fracture. A new proof for the relation between ERR and interface tractions at an unbroken interface point, not necessarily the crack tip point, was introduced. The representative nature of a thin, elastic adhesive layer by this LEBIM has been briefly discussed. The application of several energy and stress based fracture criteria in the framework of this LEBIM in a new unified form has been introduced and these criteria have been carefully analysed.

The LEBIM developed was used to characterize the onset and growth of the debond for a single fibre embedded in an infinite matrix subjected to biaxial transverse loads $\sigma_{x}^{\infty} \geq \sigma_{y}^{\infty}$, Fig. 5. Both analytic and numerical procedures were devised and exploited to study this problem. The analytical procedure was used in the parametric studies regarding debond onset and for testing the numerical procedure implemented in a collocation BEM code. The very accurate and efficient numerical procedure, however, is quite general and is currently applied to the numerical analysis of debond onset and growth in dense fibre packing representing a portion of a real unidirectional composite lamina, with several fibres, under biaxial transverse loads (Távara et al 2013). 
A comprehensive parametric study of this single-fibre debond problem analysing the influence of all the dimensionless parameters governing the problem: $\chi$ - load biaxiality (26), $\xi$ - ratio of the interface shear and normal stiffnesses (16), $\lambda$ sensitivity to the interface fracture mode mixity (18), and $\gamma$ - brittleness number (27), in addition to the elastic properties of fibre and matrix, was carried out. To the best knowledge of the authors no similar parametric study has been presented before, neither for the LEBIM nor CZMs.

Using a general analytical solution for tractions, at the undamaged linearelastic fibre-matrix interface under uniform far-field biaxial transverse stresses, and assuming the LEBIM, fairly universal failure curves on the plane of normalized far-field stresses $\left(\frac{\sigma_{x}^{\infty}}{\bar{\sigma}_{c}}, \frac{\sigma_{y}^{\infty}}{\bar{\sigma}_{c}}\right)$, where $\bar{\sigma}_{c}$ is the interface tensile strength, were generated. These curves, parameterized by $\chi$, depend only on a few dimensionless parameters $\xi, \lambda, \gamma$, and $E_{m} / E_{f}, \nu_{m}$ and $\nu_{f}$. In particular, the elastic properties $E_{m}, E_{f}, \nu_{m}$ and $\nu_{f}$ corresponding to a glass-epoxy composite were considered. It can be observed from these curves, that with decreasing $\chi$, the critical load $\sigma_{c x}^{\infty}$ decreases as well, i.e. a compression $\sigma_{y}^{\infty}$ makes crack onset easier leading to a lower critical tension load $\sigma_{c x}^{\infty}$, and vice-versa, a tension $\sigma_{y}^{\infty}$ makes a crack onset more difficult leading to a larger value for $\sigma_{c x}^{\infty}$. These observations agree with previous experimental results in (París et al 2003).

The debond onset angles $\theta_{o}(\chi)$ associated with these failure curves were also evaluated analytically. A bifurcation from the zero value of $\theta_{o}$, predicting a debond onset in mixed mode, typically occurs for a magnitude of the compression load $\sigma_{y}^{\infty}$ larger than the tension load $\sigma_{x}^{\infty}$, i.e. for $\chi<0$. Nevertheless, in very brittle configurations characterized by $\gamma \gtrsim 0$, or for interfaces with fracture energy essentially independent of the mode mixity with $\lambda \lesssim 1$, such a bifurcation can occur for small or vanishing values of $\sigma_{y}^{\infty}$.

The influence observed of the governing dimensionless parameters on the shape and location of the failure curves and the debond onset angle is summarized in the following: a) $\xi$ has only a slight influence on the shape and no influence on the position of the failure curves, also its influence on $\theta_{o}$ is quite small; b) $\lambda$ typically has no influence on the debond onset for tension dominated loads as the interface breaks at $\theta_{o}=0$ under pure mode I (except for $\gamma \gtrsim 0$ or $\lambda \lesssim 1$ ), but it has a quite big influence on $\theta_{o}$, in particular on its bifurcation point position, for compression dominated loads. Consequently $\lambda$ shows some influence on the shape of failure curves for such loads, particularly for $\lambda \gtrsim 0.5$; c) $\gamma$ has a strong influence on the position of failure curves for tough configurations $(\gamma \gtrsim 1)$, while for brittle configurations its influence on the position of failure curves is rather weak, but showing, however, some influence on their shape. The influence of $\gamma$ on $\theta_{0}$ is quite relevant for $\gamma \gtrsim 0$.

From the numerical results obtained, it can be observed that when the remote load reaches its critical value given by $\sigma_{c x}^{\infty}$, the subsequent debond growth up to the critical semidebond angle $\theta_{c}$ is unstable, an instability phenomenon called snapback which takes place under both displacement and load control. A parametric study shows that $\theta_{c}$ increases with increasing $\chi$ in the range studied, eventually very large debonds with $\theta_{c}>\frac{\pi}{2}$ are predicted when similar tensions are applied in both directions.

From the above original analytical and numerical results it appears that the new LEBIM formulation introduced adequately describes the behavior of the fibre- 
matrix system, predicting expected behaviour where some experimental results are available (París et al 2003) and also being in quite good agrement with other analytical and numerical studies (París et al 2007; Correa et al 2008b,a; Mantič and García 2012; Correa et al 2013; García et al 2014).

It has been shown that the present LEBIM implementation in a BEM code is an efficient computational tool for an interface crack onset and mixed mode crack growth modeling. This tool can be useful not only for an analysis of fibre-matrix debonding under biaxial transverse loads as carried out in the present study and in (Távara et al 2013), but also in other problems such as interlaminar fracture toughness tests of symmetric and non-symmetric laminates and delaminations in cross-ply laminates.

Acknowledgements The study was supported by the Junta de Andalucía (Projects of Excellence TEP-2045, TEP-4051 and P12-TEP-1050), The Spanish Ministry of Education and Science (Projects TRA2006-08077 and MAT2009-14022) and Spanish Ministry of Economy and Competitiveness (Projects MAT2012-37387 and DPI2012-37187).

\section{References}

Adams RD, Comyn J, Wake WC (1984) Structural Adhesive Joints in Engineering. Chapman and Hall: London

Antipov YA, Avila-Pozos O, Kolaczkowski ST, Movchan AB (2001) Mathematical model of delamination cracks on imperfect interfaces. International Journal of Solids and Structures 38:6665-6697

Bank-Sills L, Ashkenazi D (2000) A note on fracture criteria for interface fracture. International Journal of Fracture 103:177-188

Bank-Sills L, Salganik R (1994) An asymptotic approach applied to a longitudinal crack in an adhesive layer. International Journal of Fracture 68:55-73

Bažant Z, Cedolin L (1991) Stability of Structures Elastic, Inelastic, Fracture and Damage Theories. Oxford University Press: New York

Bennati S, Colleluori M, Corigliano D, Valvo PS (2009) An enhanced beam-theory model of the asymmetric double cantilever beam (ADCB) test for composite laminates. Composites Science and Technology 69:1735-1745

Benveniste Y, Miloh T (2001) Imperfect soft and stiff interfaces in two-dimensional elasticity. Mechanics of Materials 33:309-323

Benzeggagh M, Kenane M (1996) Measurement of mixed-mode delamination fracture toughness of unidirectional glass/epoxy composites with mixed mode bending apparatus. Composites Science and Technology 49:439-49

Bialas M, Mróz Z (2005a) Damage modelling at material interfaces. In: Sadowski T (ed) Multiscale Modelling of Damage and Fracture Processes in Composite Materials, Springer, Wien, pp 213-270

Bialas M, Mróz Z (2005b) Modelling of progressive interface failure under combined normal compression and shear stress. International Journal of Solids and Structures 42:4436-4467

Bigoni D, Serkov SK, Valentini M, Movchan AB (1998) Asymptotic models of dilute composites with imperfectly bonded inclusions. International Journal of Solids and Structures 35:32393258

Blázquez A, Mantič V, París F, Cañas J (1996) On the removal of rigid body motions in the solution of elastostatic problems by direct BEM. International Journal for Numerical Methods in Engineering 39:4021-4038

Brewer JC, Lagace A (1988) Quadratic stress criterion for initiation of delamination. Journal of Composite Materials : 1141 22:1141-1155

Bruno D, Greco F (2001) Mixed mode delamination in plates: a refined approach. International Journal of Solids and Structures 38:9149-9177

Bruno D, Greco F, Lonetti P (2003) A coupled interface-multilayer approach for mixed mode delamination and contact analysis in laminated composites. International Journal of Solids and Structures 40:7245-7268 
Camanho PP, Dávila CG, de Moura MF (2003) Numerical simulation of mixed-mode progressive delamination in composite materials. Journal of Composite Materials 37:1415-1438

Caporale A, Luciano R, Sacco E (2006) Micromechanical analysis of interfacial debonding in unidirectional fiber-reinforced composites. Computers \& Structures 84:2200-2211

Carpinteri A (1989) Cusp catastrophe interpretation of fracture instability. Journal of the Mechanics and Physics of Solids 37:567-582

Carpinteri A, Paggi M, Zavarise G (2005) Snap-back instability in micro-structured composites and its connection with superplasticity. Strength, Fracture and Complexity 3:61-72

Carpinteri A, Cornetti P, Pugno N (2009) Edge debonding in FRP strengthened beams: Stress versus energy failure criteria. Engineering Structures 31:2436-2447

Cornetti P, Mantič V, Carpinteri A (2012) Finite Fracture Mechanics at elastic interfaces. International Journal of Solids and Structures 49:1022-1032

Cornetti P, Corrado M, de Lorenzis L, Carpinteri A (2015) An analytical cohesive crack modeling approach to the edge debonding failure of frp-plated beams. International Journal of Solids and Structures 53:92-106

Correa E, Gamstedt EK, París F, Mantič V (2007) Effects of the presence of compression in transverse cyclic loading on fibre--matrix debonding in unidirectional composite plies. Composites Part A: Applied Science and Manufacturing 38:2260-2269

Correa E, Mantič V, París F (2008a) A micromechanical view of inter-fibre failure of composite materials under compression transverse to the fibres. Composites Science and Technology 68:2010-2021

Correa E, Mantič V, París F (2008b) Numerical characterisation of the fibre-matrix interface crack growth in composites under transverse compression. Engineering Fracture Mechanics 75:4085-4103

Correa E, París F, Mantič V (2013) Effect of the presence of a secondary transverse load on the inter-fibre failure under tension. Engineering Fracture Mechanics DOI 10.1016/j.engfracmech.2013.02.026

Ducept F, Gamby D, Davies P (1999) A mixed-mode failure criterion derived from tests on symmetric and asymmetric specimens. Composites Science and Technology 59:609-619

Entov VM, Salganik RL (1968) On the Prandtl brittle fracture model. Mechanics of Solids (translated from Russian) 3(6):79-89

Erdogan F (1997) Fracture mechanics of interfaces. In: Rossmanith HP (ed) Damage and Failure of Interfaces, A A Balkema Publishers, Rotterdam, pp 3-36

Evans AG, Rühle M, Dalgleish BJ, Charalambides PG (1990) The fracture energy of bimaterial interfaces. Metallurgical Transactions A 21:2419-2429

Fernlund G, Spelt JK (1991) Analytical method for calculating adhesive joint fracture parameters. Engineering Fracture Mechanics 40:119-132

Fiedler B, Hojo M, Ochiai S, Schulte K, Ando M (2001) Failure behavior of an epoxy matrix under different kinds of static loading. Composites Science and Technology 61:1615-1624

Gao Z (1995) A circular inclusion with imperfect interface: Eshelby's tensor and related problems. Journal of Applied Mechanics 62:860-866

García IG, Leguillon D (2012) Mixed-mode crack initiation at a v-notch in presence of an adhesive joint. International Journal of Solids and Structures 49(15-16):2138 - 2149

García IG, Paggi M, Mantič V (2014) Fiber-size effects on the onset of fiber-matrix debonding under transverse tension: A comparison between cohesive zone and finite fracture mechanics models. Engineering Fracture Mechanics 115:96-110

García IG, Mantič V, Graciani E (2015) A model for the prediction of debond onset in sphericalparticle-reinforced composites under tension. application of a coupled stress and energy criterion. Composites Science and Technology 106:60-67

Geymonat G, Krasucki F, Lenci S (1999) Mathematical analysis of a bonded joint with a soft thin adhesive. Mathematics and Mechanics of Solids 4:201-225

Goland M, Reissner E (1944) The stresses in cemented joints. Journal of Applied Mechanics 11:A17-A27

Goutianos S, Sørensen BF (2012) Path dependence of truss-like mixed mode cohesive laws. Engineering Fracture Mechanics 91:117-132

Graciani E, Mantič V, París F, Blázquez A (2005) Weak formulation of axi-symmetric frictionless contact problems with boundary elements: Application to interface cracks. Computer and Structures 83:836-855

Han R, Ingber MS, Schreyer HL (2006) Progression of failure in fiber-reinforced materials. Computers Materials \& Continua 4:163-176 
Harper PW, Sun L, Hallett SR (2012) A study on the influence of cohesive zone interface element strength parameters on mixed mode behaviour. Composites: Part A 43:722-734

Hashin Z (2002) Thin interphase/imperfect interface in elasticity with application to coated fiber composites. Journal of the Mechanics and Physics of Solids 50:2509-2537

Hull D, Clyne TW (1996) An Introduction to Composite Materials, 2nd edn. Cambridge University Press

Hutchinson JW, Suo Z (1992) Mixed mode cracking in layered materials. Advances in Applied Mechanics 29:63-191

Irwin G (1957) Analysis of stresses and strains near the end of a crack traversing a plate. Journal of Applied Mechanics 24:361-364

Kinloch AJ (1987) Adhesion and Adhesives. Science and Technology. Chapman and Hall: London

Krenk S (1992) Energy release rate of symmetric adhesive joints. Engineering Fracture Mechanics 43(4):549-559

Kushch VI, Shmegera SV, Brøndsted P, Mishnaevsky L (2011) Numerical simulation of progressive debonding in fiber reinforced composite under transverse loading. International Journal of Engineering Science 49:17-29

Kšiñan J, Mantič V, Vodička R (2014) A new interface damage model with frictional contact. an SGBEM formulation and implementation. In: Mallardo V, Aliabadi MH (eds) Advances in Boundary Element Techniques \& Meshless Techniques XV, EC Ltd, Eastleight, UK, pp 60-67

Leguillon D (2002) Strength or toughness? A criterion for crack onset at a notch. European Journal of Mechanics A/Solids 21:61-72

Lemaitre J, Desmorat R (2005) Engineering Damage Mechanics. Springer-Verlag: Berlin

Lenci S (2001) Analysis of a crack at a weak interface. International Journal of Fracture 108:275-290

Liechti KM (2002) Fracture mechanics and singularities in bonded systems. In: Dillard DA, Pocius AV (eds) The Mechanics of Adhesion, Elsevier, Amsterdam, pp 45-75

Mantič V (2009) Interface crack onset at a circular cylindrical inclusion under a remote transverse tension. Application of a coupled stress and energy criterion. International Journal of Solids and Structures 46:1287-1304

Mantič V, García IG (2012) Crack onset and growth at the fibre-matrix interface under remote biaxial transverse loads. Application of a coupled stress and energy criterion. International Journal of Solids and Structures 49:2273-2290

Mantič V, Blázquez A, Correa E, París F (2006) Analysis of interface cracks with contact in composites by 2D BEM. In: Guagliano M, Aliabadi MH (eds) Fracture and Damage of Composites, WIT Press, Southampton, pp 189-248

Mishuris GS, Kuhn G (2001) Asymptotic behaviour of the elastic solution near the tip of a crack situated at a nonideal interface. ZAMM - Zeitschrift für Angewandte Mathematik und Mechanik 81:811826

Mogilevskaya SG, Crouch SL (2002) A Galerkin boundary integral method for multiple circular elastic inclusions with homogeneously imperfect interfaces. International Journal of Solids and Structures 39:4723-4746

Mott NF (1948) Fracture of metals: Theoretical considerations. Engineering pp 16-18

Muñoz-Reja M, Távara L, Mantič V, Cornetti P (2014) Crack onset and propagation in composite materials using finite fracture mechanics on elastic interfaces. Procedia Materials Science 3:1365-1370

Ngo D, Park K, Paulino GH, Huang Y (2010) On the constitutive relation of materials with microstructure using a potential-based cohesive model for interface interaction. Engineerign Fracture Mechanics 77:1153-1174

Panagiotopoulos C, Mantič V, Roubíček T (2013) BEM implementation of energetic solutions for quasistatic delamination problems. Computational Mechanics 51:505-521

París F, Correa E, Cañas J (2003) Micromechanical view of failure of the matrix in fibrous composite materials. Composites Science and Technology 63:1041-1052

París F, Correa E, Mantič V (2007) Kinking of transverse interface cracks between fiber and matrix. Journal of Applied Mechanics 74:703-716

Pinho ST, Ianucci L, Robinson P (2006) Formulation and implementation of decohesion elements in an explicit finite element code. Composites Part A 37:778-789

Prandtl L (1933) Ein Gedankenmodell für den Zerreißvorgang spröder Körper (A thought model for the fracture of brittle solids). Zeitschrift für Angenwandte Mathematik und 
Mechanik 13(2):129-133

Raous M (2011) Interface models coupling adhesion and friction. Comptes Rendus Mecanique 339:491-501

Roubíček T, Mantič V, Panagiotopoulos C (2013) Quasistatic mixed-mode delamination model. Discrete and Continuous Dynamical Systems - Series S 6:591-610

Shahin K, Taheri F (2008) The strain energy release rates in adhesively bonded balanced and unbalanced specimens and lap joints. International Journal of Solids and Structures 45:6284-6300

Soden PD, Hinton MJ, Kaddour AS (1998) Lamina properties, lay-up configurations and loading conditions for a range of fibre-reinforced composite laminates. Composites Science and Technology 58:1011-1022

Swadener JG, Liechti KM, de Lozanne AL (1999) The intrinsic toughness and adhesion mechanisms of a glass/epoxy interface. Journal of Mechanics and Physics of Solids 47:223-258

Távara L (2010) Damage initiation and propagation in composite materials. Boundary element analysis using weak interface and cohesive zone models. PhD Thesis, Universidad de Sevilla: Sevilla

Távara L, Mantič V, Graciani E, Cañas J, París F (2010) Analysis of a crack in a thin adhesive layer between orthotropic materials. An application to composite interlaminar fracture toughness test. Computer Modeling in Engineering and Sciences 58(3):247-270

Távara L, Mantič V, Graciani E, París F (2011) BEM analysis of crack onset and propagation along fiber-matrix interface under transverse tension using a linear elastic-brittle interface model. Engineering Analysis with Boundary Elements 35:207-222

Távara L, Mantič V, Graciani E, París F (2013) BEM modelling of interface cracks in a group of fibres under biaxial transverse loads. In: Sellier A, Aliabadi MH (eds) Advances in Boundary Element Techniques XIV, Proceedings of the 14th International Conference Paris, France, EC Ltd, Eastleight, UK, pp 311-316

Thouless MD, Yang QD (2002) Measurement and analysis of the fracture properties of adhesive joints. In: Dillard DA, Pocius AV (eds) The Mechanics of Adhesion, Elsevier, Amsterdam, pp 235-271

Tvergaard V (2001) Resistance curves for mixed mode interface crack growth between dissimilar elasti-plastic solids. Journal of the Mechanics and Physics of Solids 49:2689-2703

Valoroso N, Champaney L (2006) A damage-mechanics-based approach for modelling decohesion in adhesively bonded assemblies. Engineering Fracture Mechanics 73:2774-2801

Varna J, Berglund LA, Ericson ML (1997) Transverse single fiber test for interfacial debonding in composites 2: Modelling. Composites Part A: Applied Science and Manufacturing 28:317-326

Vodička R, Mantič V, Roubíček T (2014) Energetic versus maximally-dissipative local solutions of a quasi-static rate-independent mixed-mode delamination model. Meccanica 49:29332963

Wang JS, Suo Z (1990) Experimental determination of interfacial toughness curves using Brazil-nut sandwiches. Acta Metall Mater 38:1279-1290

Wang JT (2013) Investigating some technical issues on cohesive zone modeling of fracture. Journal of Engineering Materials and Technology 135:011,003-1-10

Weißgraeber P, Becker W (2013) Finite fracture mechanics model for mixed mode fracture in adhesive joints. International Journal of Solids and Structures 50:2383-2394

Xie M, Levy A (2007) Defect propagation at a circular interface. International Journal of Fracture 144:1-20

Zhang H, Ericson ML, Varna J, Berglund LA (1997) Transverse single-fiber test for interfacial debonding in composites: 1. Experimental observations. Composites Part A: Applied Science and Manufacturing 28A:309-315 